1

2

\title{
RDF2Vec: RDF graph embeddings and their applications
}

\author{
Petar Ristoski ${ }^{\text {a, }}$, Jessica Rosati ${ }^{\text {b,c }}$, Tommaso Di Noia ${ }^{\mathrm{b}}$, Renato De Leone ${ }^{\mathrm{c}}$ and Heiko Paulheim ${ }^{\mathrm{a}}$ \\ a Data and Web Science Group, University of Mannheim, B6, 26, 68159, Mannheim \\ E-mails: petar.ristoski@informatik.uni-mannheim.de,heiko@informatik.uni-mannheim.de \\ ${ }^{\mathrm{b}}$ Polytechnic University of Bari, Via Orabona, 4, 70125, Bari \\ E-mails: jessica.rosati@poliba.it,tommaso.dinoia@poliba.it \\ ${ }^{c}$ University of Camerino, Piazza Cavour 19/f, 62032, Camerino \\ E-mails: jessica.rosati@unicam.it,renato.deleone@unicam.it
}

Editor: Freddy Lecue, Accenture Tech Labs, Ireland and INRIA, France

Solicited reviews: Achim Rettinger, Karlsruhe Institute of Technology, Germany; Jiewen Wu, Institute for Infocomm Research, Singapore; one anonymous reviewer

\begin{abstract}
Linked Open Data has been recognized as a valuable source for background information in many data mining and information retrieval tasks. However, most of the existing tools require features in propositional form, i.e., a vector of nominal or numerical features associated with an instance, while Linked Open Data sources are graphs by nature. In this paper, we present RDF2Vec, an approach that uses language modeling approaches for unsupervised feature extraction from sequences of words, and adapts them to RDF graphs. We generate sequences by leveraging local information from graph sub-structures, harvested by Weisfeiler-Lehman Subtree RDF Graph Kernels and graph walks, and learn latent numerical representations of entities in RDF graphs. We evaluate our approach on three different tasks: (i) standard machine learning tasks, (ii) entity and document modeling, and (iii) content-based recommender systems. The evaluation shows that the proposed entity embeddings outperform existing techniques, and that pre-computed feature vector representations of general knowledge graphs such as DBpedia and Wikidata can be easily reused for different tasks.
\end{abstract}

Keywords: Graph embeddings, linked open data, data mining, document semantic similarity, entity relatedness, recommender systems

\section{Introduction}

Since its introduction, the Linked Open Data (LOD) [81] initiative has played a leading role in the rise of a new breed of open and interlinked knowledge bases freely accessible on the Web, each of them being part of a huge decentralized data space, the LOD cloud. This latter is implemented as an open, interlinked collection of datasets provided in machine-interpretable form, mainly built on top of World Wide Web Consor-

$\begin{array}{cc}{ }^{*} \text { Corresponding } & \text { author. }\end{array}$

tium (W3C) standards, such as $\mathrm{RDF}^{1}$ and SPARQL. ${ }^{2}$ Currently, the LOD cloud consists of about 1,000 interlinked datasets covering multiple domains from life science to government data [81]. The LOD cloud has been recognized as a valuable source of background knowledge in data mining and knowledge discovery in general [77], as well as for information retrieval and recommender systems [20]. Augmenting a dataset with background knowledge from Linked Open Data

\footnotetext{
${ }^{1}$ http://www.w3.org/TR/2004/REC-rdf-concepts-20040210/, 2004.

${ }^{2}$ http://www.w3.org/TR/rdf-sparql-query/, 2008.
} 
can, in many cases, improve the results of the problem at hand, while externalizing the cost of maintaining that background knowledge [63].

Most data mining algorithms work with a propositional feature vector representation of the data, which means that each instance is represented as a vector of features $\left\langle f_{1}, f_{2}, \ldots, f_{n}\right\rangle$, where the features are either binary (i.e., $f_{i} \in\{$ true, false $\}$ ), numerical (i.e., $f_{i} \in \mathbb{R}$ ), or nominal (i.e., $f_{i} \in S$, where $S$ is a finite set of symbols). Linked Open Data, however, comes in the form of graphs, connecting resources with types and relations, backed by a schema or ontology. Therefore, in order to make LOD accessible to existing data mining tools, an initial propositionalization [36] of the corresponding graph is required. Even though the new set of propositional features do not encode all the knowledge available in the original ontological data, they can be effectively used to train a model via machine learning techniques and algorithms. Usually, binary features (e.g., true if a type or relation exists, false otherwise) or numerical features (e.g., counting the number of relations of a certain type) are used [65,75]. Other variants, e.g., counting different graph sub-structures, have also been proposed and used [17].

Typically, those strategies led to high-dimensional, sparse datasets, which cause problems for many data mining algorithms [1]. Therefore, dense, lowerdimensional representations are usually preferred, but less straight forward to generate.

In language modeling, vector space word embeddings have been proposed in 2013 by Mikolov et al. $[43,44]$. They train neural networks for creating a lowdimensional, dense representation of words, which show two essential properties: (a) similar words are close in the vector space, and (b) relations between pairs of words can be represented as vectors as well, allowing for arithmetic operations in the vector space. In this work, we adapt those language modeling approaches for creating a latent representation of entities in RDF graphs.

Since language modeling techniques work on sentences, we first convert the graph into a set of sequences of entities using two different approaches, i.e., graph walks and Weisfeiler-Lehman Subtree RDF graph kernels. In the second step, we use those sequences to train a neural language model, which estimates the likelihood of a sequence of entities appearing in a graph. Once the training is finished, each entity in the graph is represented as a vector of latent numerical features. We show that the properties of word embeddings also hold for RDF entity embeddings, and that they can be exploited for various tasks.

We use several RDF graphs to show that such latent representation of entities have high relevance for different data mining and information retrieval tasks. The generation of the entities' vectors is task and dataset independent, i.e., we show that once the vectors are generated, they can be reused for machine learning tasks, like classification and regression, entity and document modeling, and to estimate the proximity of items for content-based or hybrid recommender systems. Furthermore, since all entities are represented in a low dimensional feature space, building the learning models and algorithms becomes more efficient. To foster the reuse of the created feature sets, we provide the vector representations of DBpedia and Wikidata entities as ready-to-use files for download.

This paper considerably extends [76], in which we introduced RDF2 Vec for the first time. In particular, we demonstrate the versatility of RDF embeddings by extending the experiments to a larger variety of tasks: we show that the vector embeddings not only can be used in machine learning tasks, but also for document modeling and recommender systems, without a need to retrain the embedding models. In addition, we extend the evaluation section for the machine learning tasks by comparing our proposed approach to some of the state-of-the-art graph embeddings, which have not been used for the specified tasks before. In further sets of experiments, we compare our approach to the state-of-the-art entity relatedness and document modeling approaches, as well to the state-of-the-art graph embedding approaches.

Preliminary results for the recommender task have already been published in [78]. In this paper, we further extend the evaluation in recommendation scenarios by considering a new dataset (Last.FM) and by implementing a hybrid approach based on Factorization Machines. Both contributions highlight the effectiveness of RDF2 Vec in building recommendation engines that overcame state of the art hybrid approaches in terms of accuracy also when dealing with very sparse datasets (as is the case of Last. FM).

The rest of this paper is structured as follows. In Section 2, we give an overview of related work. In Section 3, we introduce our approach. In Section 4 through Section 7, we describe the evaluation setup and evaluate our approach on three different sets of tasks, i.e., machine learning, document modeling, and recommender systems. We conclude with a summary and an outlook on future work. 


\section{Related work}

While the representation of RDF as vectors in an embedding space itself is a considerably new area of research, there is a larger body of related work in the three application areas discussed in this paper, i.e., the use of LOD in data mining, in document modeling, and in content-based recommender systems.

Generally, our work is closely related to the approaches DeepWalk [67] and Deep Graph Kernels [95]. DeepWalk uses language modeling approaches to learn social representations of vertices of graphs by modeling short random-walks on large social graphs, like BlogCatalog, Flickr, and YouTube. The Deep Graph Kernel approach extends the DeepWalk approach by modeling graph substructures, like graphlets, instead of random walks. Node2vec [24] is another approach very similar to DeepWalk, which uses second order random walks to preserve the network neighborhood of the nodes. The approach we propose in this paper differs from these approaches in several aspects. First, we adapt the language modeling approaches on directed labeled RDF graphs, unlike the approaches mentioned above, which work on undirected graphs. Second, we show that task-independent entity vectors can be generated on large-scale knowledge graphs, which later can be reused on a variety of machine learning tasks on different datasets.

\subsection{LOD in machine learning}

In the recent past, a few approaches for generating data mining features from Linked Open Data have been proposed. Many of those approaches assume a manual design of the procedure for feature selection and, in most cases, this procedure results in the formulation of a SPARQL query by the user. LiDDM [50] allows the users to declare SPARQL queries for retrieving features from LOD that can be used in different machine learning techniques. Similarly, Cheng et al. [10] proposes an approach for automated feature generation after the user has specified the type of features in the form of custom SPARQL queries.

A similar approach has been used in the RapidMiner $^{3}$ semweb plugin [33], which preprocesses RDF data in a way that can be further handled directly in RapidMiner. Mynarz et al. [49] have considered using user specified SPARQL queries in combination with

\footnotetext{
${ }^{3}$ http://www.rapidminer.com/
}

SPARQL aggregates. FeGeLOD [65] and its successor, the RapidMiner Linked Open Data Extension [72], have been the first fully automatic unsupervised approach for enriching data with features that are derived from LOD. The approach uses six different unsupervised feature generation strategies, exploring specific or generic relations. It has been shown that such feature generation strategies can be used in many data mining tasks [66,72].

When dealing with Kernel Functions for graphbased data, we face similar problems as in feature generation and selection. Usually, the basic idea behind their computation is to evaluate the distance between two data instances by counting common substructures in the graphs of the instances, i.e., walks, paths and trees. In the past, many graph kernels have been proposed that are tailored towards specific applications [31,59], or towards specific semantic representations [13]. However, only a few approaches are general enough to be applied on any given RDF data, regardless the data mining task. Lösch et al. [41] introduce two general RDF graph kernels, based on intersection graphs and intersection trees. Later, the intersection tree path kernel was simplified by de Vries et al. [16]. In another work, de Vries et al. [15,17] introduce an approximation of the state-of-the-art WeisfeilerLehman graph kernel algorithm aimed at improving the computation time of the kernel when applied to RDF. Furthermore, the kernel implementation allows for explicit calculation of the instances' feature vectors, instead of pairwise similarities.

Furthermore, multiple approaches for knowledge graph embeddings for the task of link prediction have been proposed [51], which could also be considered as approaches for generating propositional features from graphs. RESCAL [53] is one of the earliest approaches, which is based on factorization of a threeway tensor. The approach is later extended into Neural Tensor Networks (NTN) [85] which can be used for the same purpose. One of the most successful approaches is the model based on translating embeddings, TransE [6]. This model builds entity and relation embeddings by regarding a relation as translation from head entity to tail entity. This approach assumes that some relationships between words could be computed by their vector difference in the embedding space. However, this approach cannot deal with reflexive, one-to-many, many-to-one, and many-to-many relations. This problem was resolved in the TransH model [92], which models a relation as a hyperplane together with a translation operation on it. More precisely, each relation is 
characterized by two vectors, the norm vector of the hyperplane, and the translation vector on the hyperplane. While both TransE and TransH, embed the relations and the entities in the same semantic space, the TransR model [40] builds entity and relation embeddings in separate entity space and multiple relation spaces. This approach is able to model entities that have multiple aspects, and various relations that focus on different aspects of entities.

\subsection{Entity and document modeling}

Both for entity and document ranking, as well as for the subtask of computing the similarity or relatedness of entities and documents, different methods using LOD have been proposed.

\subsubsection{Entity relatedness}

Semantic relatedness of entities has been heavily researched over the past couple of decades. There are two main direction of studies. The first are approaches based on word distributions, which model entities as multi-dimensional vectors that are computed based on distributional semantics techniques $[2,21,29]$. The second are graph-based approaches relying on a graph structured knowledge base, or knowledge graph, which are the focus of this paper.

Schuhmacher et al. [82] proposed one of the first approaches for entity ranking using the DBpedia knowledge graph. They use several path and graph based approaches for weighting the relations between entities, which are later used to calculate the entity relatedness. A similar approach is developed by Hulpus et al. [32], which uses local graph measures, targeted to the specific pair, while the previous approach uses global measures. More precisely, the authors propose the exclusivity-based relatedness measure that gives higher weights to relations that are less used in the graph. In [56] the authors propose a hybrid approach that exploits both textual and RDF data to rank resources in DBpedia related to the IT domain.

\subsubsection{Entity and document similarity}

As for the entity relatedness approaches, there are two main directions of research in the field of semantic document similarity, i.e., approaches based on word distributions, and graph-based approaches. Some of the earliest approaches of the first category make use of standard techniques like bag-of-words models, but also more sophisticated approaches. Explicit Semantic Analysis (ESA) [21] represents text as a vector of relevant concepts. Each concept corresponds to a
Wikipedia article mapped into a vector space using the TF-IDF measure on the article's text. Similarly, Salient Semantic Analysis (SSA) [27] uses hyperlinks within Wikipedia articles to other articles as vector features, instead of using the full body of text.

Nunes et al. [58] present a DBpedia based document similarity approach, in which they compute a document connectivity score based on document annotations, using measures from social network theory. Thiagarajan et al. [88] present a general framework showing how spreading activation can be used on semantic networks to determine similarity of groups of entities. They experiment with Wordnet and the Wikipedia Ontology as knowledge bases and determine similarity of generated user profiles based on a 1-1 annotation matching.

Schuhmacher et al. [82] use the same measure used for entity ranking (see above) to calculate semantic document similarity. Similarly, Paul et al. [62] present an approach for efficient semantic similarity computation that exploits hierarchical and transverse relations in the graph.

One approach that does not belong to these two main directions of research is the machine-learning approach by Huang et al. [30]. The approach proposes a measure that assesses similarity at both the lexical and semantic levels, and learns from human judgments how to combine them by using machine-learning techniques.

Our work is, to the best of our knowledge, the first to exploit the graph structure using neural language modeling for the purpose of entity relatedness and similarity.

\subsection{Recommender systems}

Providing accurate suggestions, tailored to user's needs and interests, is the main target of Recommender Systems (RS) [70], information filtering techniques commonly used to suggest items that are likely to be of use to a user. These techniques have proven to be very effective to face the information overload problem, that is the huge amount of information available on the Web, which risks to overwhelm user's experience while retrieving items of interest. The numerous approaches facilitate the access to information in a personalized way, building a user profile and keeping it up-to-date.

RS address the information overload issue in two different ways, often combined into hybrid systems [8]: the collaborative approach [70] exploits informa- 
tion about the past behaviour and opinions of an existing user community to predict which item the current user will be more interested in, while the contentbased approach [70] relies on the items' "content", that is the description of their characteristics. In a collaborative setting, a profile of the user is built by estimating her choice pattern through the behaviour of the overall user community. The content-based approach, instead, represents items by means of a set of features and defines a user profile as an assignment of importance to such features, exploiting the past interaction with the system. To overcome the limitations of traditional approaches, which define the content based on partial metadata or on textual information optionally associated to an item, a process of "knowledge infusion" [83] has been performed for the last years, giving rise to the class of semantics-aware contentbased recommender systems [14]. Many content-based RS have incorporated ontological knowledge [42], unstructured or semi-structured knowledge sources (e.g., Wikipedia) [83], or the wealth of the LOD cloud, and recently the interest in unsupervised techniques where the human intervention is reduced or even withdrawn, has significantly increased.

LOD datasets, e.g., DBpedia [39], have been used in content-based recommender systems, e.g., in [19] and [20]. The former performs a semantic expansion of the item content based on ontological information extracted from DBpedia and LinkedMDB [28], an open semantic web database for movies, and tries to derive implicit relations between items. The latter involves both DBpedia and LinkedMDB and adapts the Vector Space Model to Linked Open Data: it represents the RDF graph as a three-dimensional tensor, where each slice is an ontological property (e.g. starring, director, ...) and represents its adjacency matrix.

It has been proved that leveraging LOD datasets is also effective for hybrid recommender systems [8], that is in those approaches that boost the collaborative information with additional knowledge about the items. In [57], the authors propose SPRank, a hybrid recommendation algorithm that extracts semantic path-based features from DBpedia and uses them to compute top- $N$ recommendations in a learning to rank approach and in multiple domains, movies, books and musical artists. SPRank is compared with numerous collaborative approaches based on matrix factorization [35,69] and with other hybrid RS, such as $B P R$-SSLIM [55], and exhibits good performance especially in those contexts characterized by high sparsity, where the contribution of the content becomes essential. Another hybrid approach is proposed in [74], which builds on training individual base recommenders and using global popularity scores as generic recommenders. The results of the individual recommenders are combined using stacking regression and rank aggregation.

Most of these approaches can be referred to as topdown approaches [14], since they rely on the integration of external knowledge and cannot work without human intervention. On the other side, bottom-up approaches ground on the distributional hypothesis [26] for language modeling, according to which the meaning of words depends on the context in which they occur, in some textual content. The resulting strategy is therefore unsupervised, requiring a corpus of textual documents for training as large as possible. Approaches based on the distributional hypothesis, referred to as discriminative models, behave as word embedding techniques where each term (and document) becomes a point in the vector space. They substitute the term-document matrix typical of Vector Space Model with a term-context matrix, on which they apply dimensionality reduction techniques such as Latent Semantic Indexing (LSI) [18] and the more scalable and incremental Random Indexing (RI) [79]. The latter has been involved in [47] and [48] to define the so called enhanced Vector Space Model (eVSM) for content-based RS, where user's profile is incrementally built summing the features vectors representing documents liked by the user and a negation operator is introduced to take into account also negative preferences, inspired by [94], that is according to the principles of Quantum Logic.

Word embedding techniques are not limited to LSI and RI. The word2vec strategy has been recently presented in [43] and [44], and to the best of our knowldge, has been applied to item recommendations in a few works [46,61]. In particular, [46] is an empirical evaluation of LSI, RI and word2vec to make contentbased movie recommendation exploiting textual information from Wikipedia, while [61] deals with checkin venue (location) recommendations and adds a nontextual feature, the past check-ins of the user. They both draw the conclusion that word2vec techniques are promising for the recommendation task. Finally, there is a single example of product embedding [23], namely prod2vec, which operates on the artificial graph of purchases, treating a purchase sequence as a "sentence" and products within the sequence as words. 


\section{Approach}

In our approach, we adapt neural language models for RDF graph embeddings. Such approaches take advantage of the word order in text documents, explicitly modeling the assumption that closer words in a sequence are statistically more dependent. In the case of RDF graphs, we consider entities and relations between entities instead of word sequences. Thus, in order to apply such approaches on RDF graph data, we first have to transform the graph data into sequences of entities, which can be considered as sentences. Using those sentences, we can train the same neural language models to represent each entity in the RDF graph as a vector of numerical values in a latent feature space.

\subsection{RDF graph sub-structures extraction}

We propose two general approaches for converting graphs into a set of sequences of entities, i.e, graph walks and Weisfeiler-Lehman Subtree RDF Graph Kernels.

Definition 1. An RDF graph is a labeled, directed graph $G=(V, E)$, where $V$ is a set of vertices, and $E$ is a set of directed edges, where each vertex $v \in V$ is identified by a unique identifier, and each edge $e \in E$ is labeled with a label from a finite set of edge labels.

The objective of the conversion functions is for each vertex $v \in V$ to generate a set of sequences $S_{v}$, where the first token of each sequence $s \in S_{v}$ is the vertex $v$ followed by a sequence of tokens, which might be edge labels, vertex identifiers, or any substructure extracted from the RDF graph, in an order that reflects the relations between the vertex $v$ and the rest of the tokens, as well as among those tokens.

\subsubsection{Graph walks}

In this approach, given a graph $G=(V, E)$, for each vertex $v \in V$, we generate all graph walks $P_{v}$ of depth $d$ rooted in vertex $v$. To generate the walks, we use the breadth-first algorithm. In the first iteration, the algorithm generates paths by exploring the direct outgoing edges of the root node $v_{r}$. The paths generated after the first iteration will have the following pattern $v_{r} \rightarrow e_{i}$, where $e_{i} \in E_{v_{r}}$, and $E_{v_{r}}$ is the set of all outgoing edges from the root node $v_{r}$. In the second iteration, for each of the previously explored edges the algorithm visits the connected vertices. The paths generated after the second iteration will follow the following pattern $v_{r} \rightarrow e_{i} \rightarrow v_{i}$. The algorithm continues until

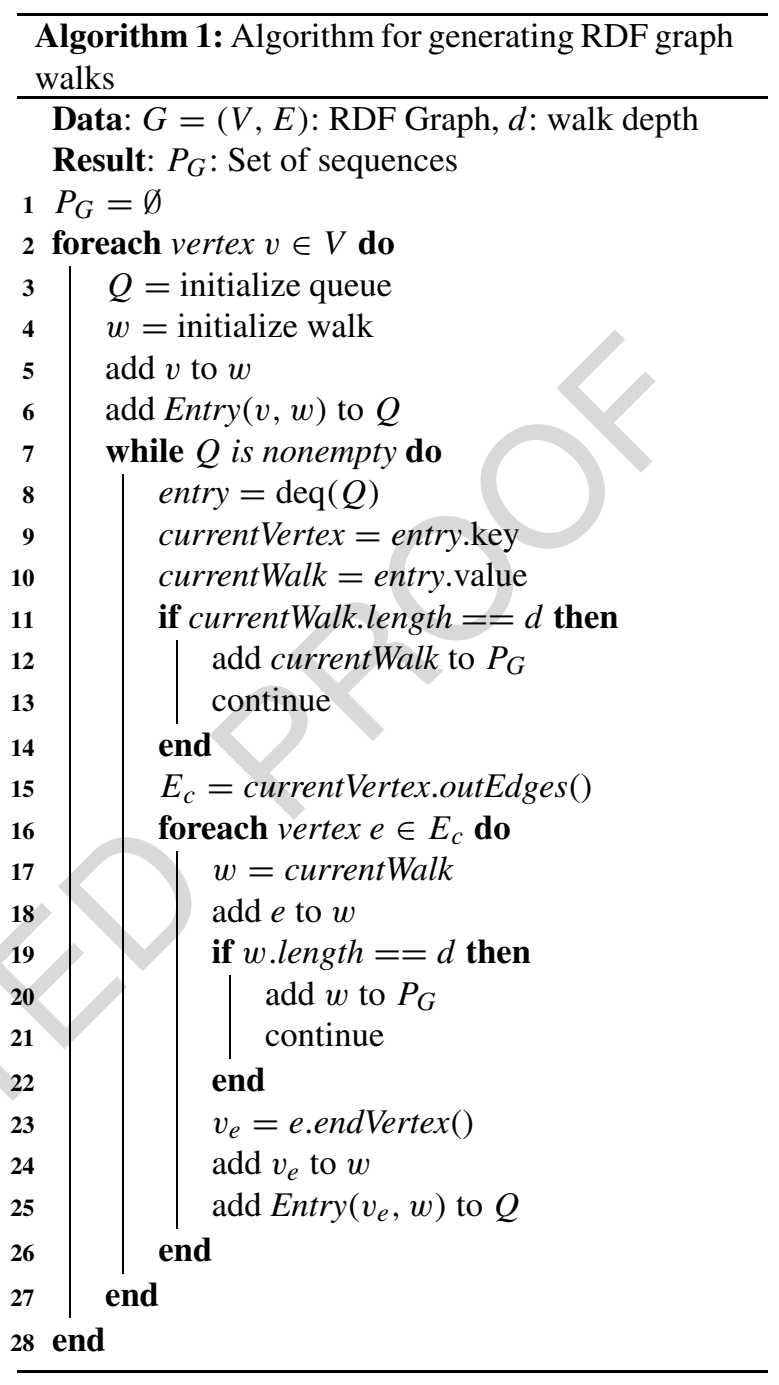

$d$ iterations are reached. The final set of sequences for the given graph $G$ is the union of the sequences of all the vertices $P_{G}=\bigcup_{v \in V} P_{v}$. The algorithm is shown in Algorithm 1.

In the case of large RDF graphs, generating all possible walks for all vertices results in a large number of walks, which makes the training of the neural language model highly inefficient. To avoid this problem, we suggest for each vertex in the graph to generate only a subset, with size $n$, of all possible walks. To generate the walks, the outgoing edge to follow from the currently observed vertex $v_{c}$ is selected based on the edge weight, i.e., the probability for selecting an edge $e_{i}$ is $\operatorname{Pr}\left[e_{i}\right]=\frac{\text { weight }\left(e_{i}\right)}{\sum_{j=1}^{\left|E v_{c}\right|} \text { weight }\left(e_{j}\right)}$, where $e_{i} \in E_{v_{c}}$, and $E_{v_{c}}$ is the set of all outgoing edges from the current node $v_{c}$. 


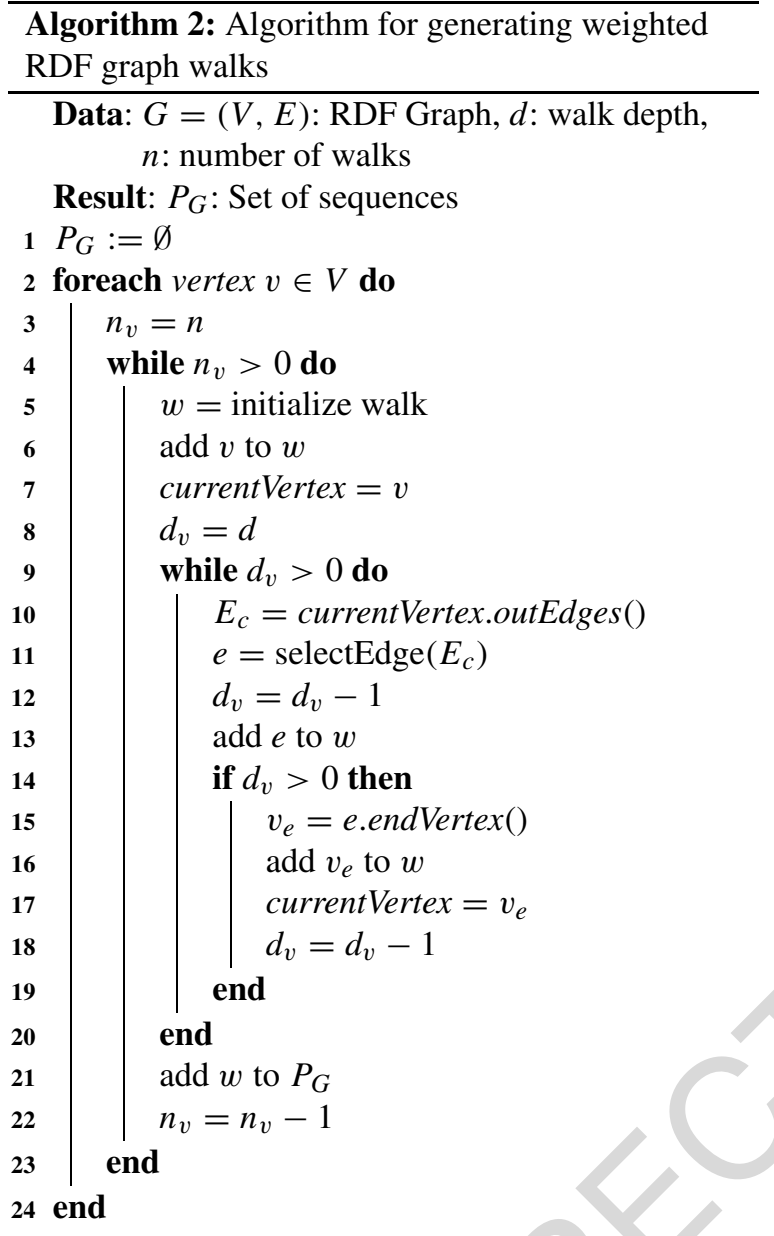

While there are many possibilities to set the weight of the edges, in this work we only consider equal weights, i.e., random selection of outgoing edges where an edge $e_{i}$ is selected with probability $\operatorname{Pr}\left[e_{i}\right]=\frac{1}{\left|E\left(v_{c}\right)\right|}$, where $e_{i} \in E_{v_{c}}$, and $E_{v_{c}}$ is the set of all outgoing edges from the current node $v_{c}$. The algorithm is shown in Algorithm 2. Other weighting strategies can be integrated into the algorithm by replacing the function selectEdge in line 11, e.g., weighting the edge based on the frequency, based on the frequency of the edge's end node, or based on global weighting metrics, like PageRank [7]. The impact of different weighting strategies on the resulting embeddings has been discussed in [11].

\subsubsection{Weisfeiler-Lehman subtree RDF graph kernels}

As an alternative to graph walks, we also use the subtree RDF adaptation of the Weisfeiler-Lehman algorithm presented in $[15,17]$ to convert an RDF graph into a set of sequences. The Weisfeiler-Lehman Sub- tree graph kernel is a state-of-the-art, efficient kernel for graph comparison [84]. The kernel computes the number of sub-trees shared between two (or more) graphs by using the Weisfeiler-Lehman test of graph isomorphism. This algorithm creates labels representing subtrees in $h$ iterations, i.e., after each iteration there is a set of subtrees, where each of the subtrees is identified with a unique label. The rewriting procedure of Weisfeiler-Lehman goes as follows: (i) the algorithm creates a multiset label for each vertex based on the labels of the neighbors of that vertex; (ii) this multiset is sorted and together with the original label concatenated into a string, which is the new label; (iii) for each unique string a new (shorter) label replaces the original vertex label; (iv) at the end of each iteration, each label represents a unique full subtree.

There are two main modifications of the original Weisfeiler-Lehman graph kernel algorithm in order to be applicable on RDF graphs [15,17]. First, the RDF graphs have directed edges, which is reflected in the fact that the neighborhood of a vertex $v$ contains only the vertices reachable via outgoing edges. Second, as mentioned in the original algorithm, labels from two iterations can potentially be different while still representing the same subtree. To make sure that this does not happen, the authors in $[15,17]$ have added tracking of the neighboring labels in the previous iteration, via the multiset of the previous iteration. If the multiset of the current iteration is identical to that of the previous iteration, the label of the previous iteration is reused.

The Weisfeiler-Lehman relabeling algorithm for an RDF graph is given in Algorithm 3, which is the same relabeling algorithm proposed in [15]. The algorithm takes as input the RDF graph $G=(V, E)$, a labeling function $l$, which returns a label of a vertex or edge in the graph based on an index, the subraph depth $d$ and the number of iterations $h$. The algorithm returns the labeling functions for each iteration $l_{0}$ to $l_{h}$, and a label dictionary $f$. Furthermore, the neighborhood $N(v)=$ $\left(v^{\prime}, v\right) \in E$ of a vertex is the set of edges going to the vertex $v$ and the neighborhood $N\left(\left(v, v^{\prime}\right)\right)=v$ of an edge is the vertex that the edge comes from.

The procedure of converting the RDF graph to a set of sequences of tokens goes as follows: (i) for a given graph $G=(V, E)$, we define the Weisfeiler-Lehman algorithm parameters, i.e., the number of iterations $h$ and the vertex subgraph depth $d$, which defines the subgraph in which the subtrees will be counted for the given vertex; (ii) after each iteration, for each vertex $v \in V$ of the original graph $G$, we extract all the paths of depth $d$ within the subgraph of the vertex $v$ 


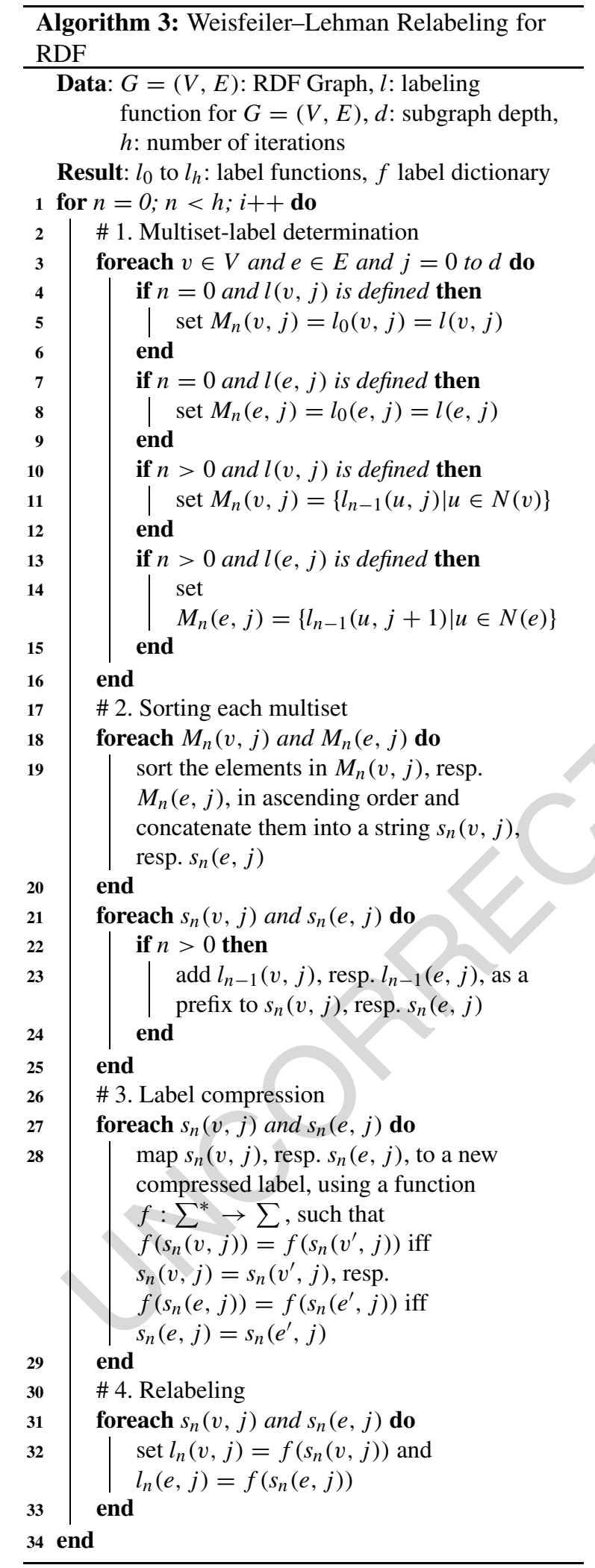

on the relabeled graph using Algorithm 1. We set the original label of the vertex $v$ as the starting token of each path, which is then considered as a sequence of tokens. The sequences after each iteration will have the following pattern $v_{r} \rightarrow l_{n}\left(e_{i}, j\right) \rightarrow l_{n}\left(v_{i}, j\right)$, where $l_{n}$ returns the label of the edges and the vertices in the $n$th iteration. The sequences could also be seen as $v_{r} \rightarrow T_{1} \rightarrow T_{1} \cdots T_{d}$, where $T_{d}$ is a subtree that appears on depth $d$ in the vertex's subgraph; (iii) we repeat step (ii) until the maximum iterations $h$ are reached. (iv) The final set of sequences is the union of the sequences of all the vertices in each iteration $P_{G}=\bigcup_{i=1}^{h} \bigcup_{v \in V} P_{v}$.

\subsection{Neural language models - word2vec}

Neural language models have been developed in the NLP field as an alternative to represent texts as a bag of words, and hence, a binary feature vector, where each vector index represents one word. While such approaches are simple and robust, they suffer from several drawbacks, e.g., high dimensionality and severe data sparsity, which limits their performance. To overcome such limitations, neural language models have been proposed, inducing low-dimensional, distributed embeddings of words by means of neural networks. The goal of such approaches is to estimate the likelihood of a specific sequence of words appearing in a corpus, explicitly modeling the assumption that closer words in the word sequence are statistically more dependent.

While some of the initially proposed approaches suffered from inefficient training of the neural network models, like Feedforward Neural Net Language Model (NNLM) [4,12,90], with the recent advances in the field several efficient approaches have been proposed. One of the most popular and widely used approaches is the word2vec neural language model $[43,44]$. Word2vec is a particularly computationallyefficient two-layer neural net model for learning word embeddings from raw text. There are two different algorithms, the Continuous Bag-of-Words model (CBOW) and the Skip-gram model. The efficiency of the models comes as a result from the simplicity of the models by avoiding dense matrix multiplication, i.e., the non-linear hidden layer is removed from the neural network and the projection layer is shared for all words. Furthermore, the Skip-gram model has been extended to make the training even more efficient, i.e., (i) sub-sampling of frequent words, which significantly improves the model training efficiency, and im- 

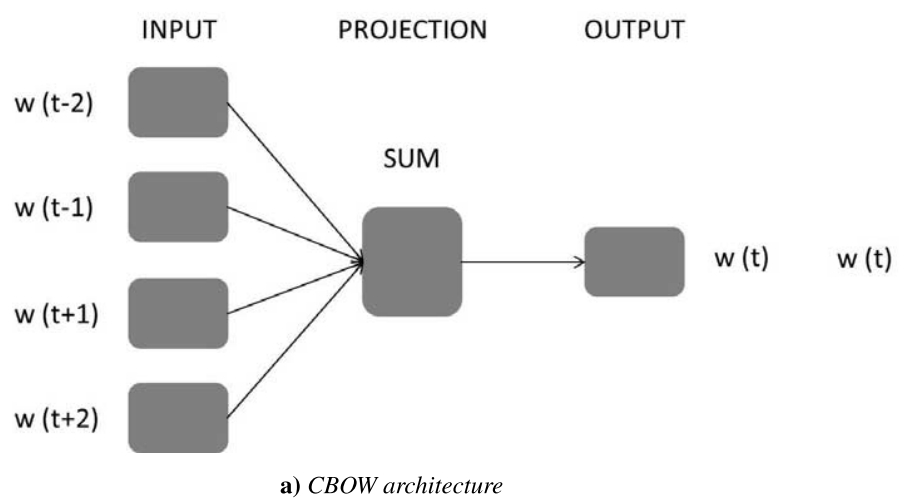

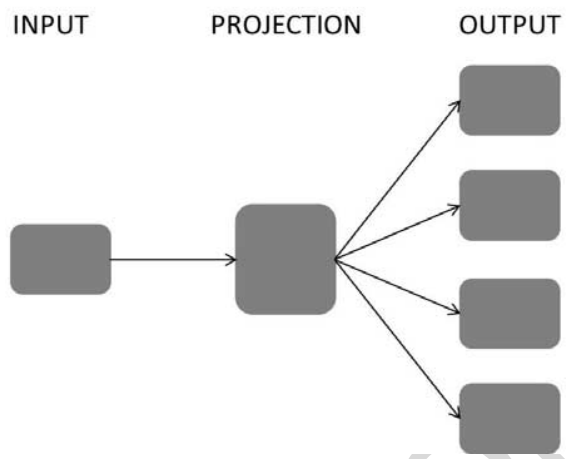

b) Skip-gram architecture
52

53

54

proves the vector quality of the less frequent words; (ii) using simplified variant of Noise Contrastive Estimation [25], called negative sampling.

\subsubsection{Continuous bag-of-words model}

The CBOW model predicts target words from context words within a given window. The model architecture is shown in Fig. 1(a). The input layer is comprised of all the surrounding words for which the input vectors are retrieved from the input weight matrix, averaged, and projected in the projection layer. Then, using the weights from the output weight matrix, a score for each word in the vocabulary is computed, which is the probability of the word being a target word. Formally, given a sequence of training words $w_{1}, w_{2}, w_{3}, \ldots, w_{T}$, and a context window $c$, the objective of the CBOW model is to maximize the average log probability:

$$
\frac{1}{T} \sum_{t=1}^{T} \log p\left(w_{t} \mid w_{t-c} \cdots w_{t+c}\right)
$$

where the probability $p\left(w_{t} \mid w_{t-c} \cdots w_{t+c}\right)$ is calculated using the softmax function:

$$
p\left(w_{t} \mid w_{t-c} \cdots w_{t+c}\right)=\frac{\exp \left(\bar{v}^{T} v_{w_{t}}^{\prime}\right)}{\sum_{w=1}^{V} \exp \left(\bar{v}^{T} v_{w}^{\prime}\right)},
$$

where $v_{w}^{\prime}$ is the output vector of the word $w, V$ is the complete vocabulary of words, and $\bar{v}$ is the averaged input vector of all the context words:

$$
\bar{v}=\frac{1}{2 c} \sum_{-c \leqslant j \leqslant c, j \neq 0} v_{w_{t+j}}
$$

\subsubsection{Skip-gram model}

The skip-gram model does the inverse of the CBOW model and tries to predict the context words from the target words (Fig. 1(b)). More formally, given a sequence of training words $w_{1}, w_{2}, w_{3}, \ldots, w_{T}$, and a context window of size $c$, the objective of the skipgram model is to maximize the following average log probability:

where the probability $p\left(w_{t+j} \mid w_{t}\right)$ is calculated using the softmax function:

$$
p\left(w_{t+c} \mid w_{t}\right)=\frac{\exp \left(v_{w_{t+c}}^{\prime T} v_{w_{t}}\right)}{\sum_{v=1}^{V} \exp \left(v_{w_{v}}^{\prime T} v_{w_{t}}\right)},
$$

where $v_{w}$ and $v_{w}^{\prime}$ are the input and the output vector of the word $w$, and $V$ is the complete vocabulary of words.

In both cases, calculating the softmax function is computationally inefficient, as the cost for computing is proportional to the size of the vocabulary. Therefore, two optimization techniques have been proposed, i.e., hierarchical softmax and negative sampling [44]. The empirical studies in the original paper [44] have shown that in most cases negative sampling leads to a better performance than hierarchical softmax, which depends on the selected negative samples, but it has higher runtime.

Once the training is finished, all words (or, in our case, entities) are projected into a lower-dimensional feature space, and semantically similar words (or entities) are positioned close to each other.

$$
\frac{1}{T} \sum_{t=1}^{T} \sum_{-c \leqslant j \leqslant c, j \neq 0} \log p\left(w_{t+j} \mid w_{t}\right)
$$




\section{Evaluation}

We evaluate our approach on three different tasks: (i) standard machine-learning classification and regression; (ii) document similarity and entity relatedness; (iii) top- $N$ recommendation both with contentbased and hybrid RSs. For all three tasks, we utilize two of the most prominent RDF knowledge graphs [64], i.e., DBpedia [39] and Wikidata [91]. DBpedia is a knowledge graph which is extracted from structured data in Wikipedia. The main source for this extraction are the key-value pairs in the Wikipedia infoboxes. Wikidata is a collaboratively edited knowledge graph, operated by the Wikimedia foundation ${ }^{4}$ which also hosts various language editions of Wikipedia.

We use the English version of the 2015-10 DBpedia dataset, which contains 4,641,890 instances and 1,369 mapping-based object properties. ${ }^{5}$ In our evaluation, we only consider object properties, and ignore datatype properties and literals.

For the Wikidata dataset, we use the simplified and derived RDF dumps from 2016-03-28. ${ }^{6}$ The dataset contains 17,340,659 entities in total. As for the DBpedia dataset, we only consider object properties, and ignore the data properties and literals.

The first step of our approach is to convert the RDF graphs into a set of sequences. As the number of generated walks increases exponentially [17] with the graph traversal depth, calculating Weisfeiler-Lehman subtrees RDF kernels, or all graph walks with a given depth $d$ for all of the entities in the large RDF graph quickly becomes unmanageable. Therefore, to extract the entities embeddings for the large RDF datasets, we use only random graph walks entity sequences, generated using Algorithm 2. For both DBpedia and Wikidata, we first experiment with 200 random walks per entity with depth of 4, and 200 dimensions for the entities' vectors. Additionally, for DBpedia we experiment with 500 random walks per entity with depth of 4 and 8, with 200 and 500 dimensions for the entities' vectors. For Wikidata, we were unable to build models with more than 200 walks per entity, because of memory constrains, therefore we only experiment with the dimensions of the entities' vectors, i.e., 200 and 500.

\footnotetext{
${ }^{4}$ http://wikimediafoundation.org/

5 http://wiki.dbpedia.org/services-resources/datasets/ dbpedia-datasets

${ }^{6}$ http://tools.wmflabs.org/wikidata-exports/rdf/index.php? content=dump_download.php\&dump=20160328
}

We use the corpora of sequences to build both CBOW and Skip-Gram models with the following parameters: window size $=5$; number of iterations $=$ 5 ; negative sampling for optimization; negative samples $=25$; with average input vector for CBOW. The parameter values are selected based on recommendations from the literature [43]. To prevent sharing the context between entities in different sequences, each sequence is considered as a separate input in the model, i.e., the sliding window restarts for each new sequence. We used the gensim implementation ${ }^{7}$ for training the models. All the models, as well as the code, are publicly available. ${ }^{8}$

In the evaluation section we use the following notation for the models: KB2Vec model \#walks \#dimensions depth, e.g. DB2vec SG 200w 200v 4d, refers to a model built on DBpedia using the skip-gram model, with 200 walks per entity, 200 dimensional vectors and all the walks are of depth 4.

\section{Machine learning with background knowledge from LOD}

Linking entities in a machine learning task to those in the LOD cloud helps generating additional features, which may help improving the overall learning outcome. For example, when learning a predictive model for the success of a movie, adding knowledge from the LOD cloud (such as the movie's budget, director, genre, Oscars won by the starring actors, etc.) can lead to a more accurate model.

\subsection{Experimental setup}

For evaluating the performance of our RDF embeddings in machine learning tasks, we perform an evaluation on a set of benchmark datasets. The dataset contains four smaller-scale RDF datasets (i.e., AIFB, MUTAG, BGS, and AM), where the classification target is the value of a selected property within the dataset, and five larger datasets linked to DBpedia and Wikidata, where the target is an external variable (e.g., the metacritic score of an album or a movie). The latter datasets are used both for classification and regression. Details on the datasets can be found in [73].

For each of the small RDF datasets, we first build two corpora of sequences, i.e., the set of sequences

${ }^{7}$ https://radimrehurek.com/gensim/

${ }^{8}$ http://data.dws.informatik.uni-mannheim.de/rdf2vec/ 
generated from graph walks with depth 8 (marked as $\mathrm{W} 2 \mathrm{~V}$ ), and set of sequences generated from Weisfeiler-Lehman subtree kernels (marked as K2V). For the Weisfeiler-Lehman algorithm, we use 3 iterations and depth of 4 , and after each iteration we extract all walks for each entity with the same depth. We use the corpora of sequences to build both CBOW and Skip-Gram models with the following parameters: window size $=5$; number of iterations $=10$; negative sampling for optimization; negative samples $=25$; with average input vector for CBOW. We experiment with 200 and 500 dimensions for the entities' vectors.

We use the RDF embeddings of DBpedia and Wikidata (see Section 4) on the five larger datasets, which provide classification/regression targets for DBpedia/Wikidata entities (see Table 1).

We compare our approach to several baselines. For generating the data mining features, we use three strategies that take into account the direct relations to other resources in the graph $[65,75]$, and two strategies for features derived from graph sub-structures [17]:

- Features derived from specific relations. In the experiments we use the relations rdf:type (types), and dcterms:subject (categories) for datasets linked to DBpedia.

- Features derived from generic relations, i.e., we generate a feature for each incoming (rel in) or outgoing relation (rel out) of an entity, ignoring the value or target entity of the relation. Furthermore, we combine both incoming and outgoing relations (rel in \& out).

- Features derived from generic relations-values, i.e., we generate a feature for each incoming (rel-

\section{Table 1}

Datasets overview. For each dataset, we depict the number of instances, the machine learning tasks in which the dataset is used $(C$ stands for classification, and $R$ stands for regression) and the source of the dataset. In case of classification, $c$ indicates the number of classes

\begin{tabular}{lccl}
\hline Dataset & \#Instances & ML Task & Original Source \\
\hline Cities & 212 & $\mathrm{R} / \mathrm{C}(c=3)$ & Mercer \\
Metacritic Albums & 1600 & $\mathrm{R} / \mathrm{C}(c=2)$ & Metacritic \\
Metacritic Movies & 2000 & $\mathrm{R} / \mathrm{C}(c=2)$ & Metacritic \\
AAUP & 960 & $\mathrm{R} / \mathrm{C}(c=3)$ & JSE \\
Forbes & 1585 & $\mathrm{R} / \mathrm{C}(c=3)$ & Forbes \\
AIFB & 176 & $\mathrm{C}(c=4)$ & AIFB \\
MUTAG & 340 & $\mathrm{C}(c=2)$ & MUTAG \\
BGS & 146 & $\mathrm{C}(c=2)$ & BGS \\
AM & 1000 & $\mathrm{C}(c=11)$ & AM \\
\hline
\end{tabular}

vals in) or outgoing relation (rel-vals out) of an entity including the value of the relation. Furthermore, we combine both incoming and outgoing relations with the values (rel-vals in \& out).

- Kernels that count substructures in the RDF graph around the instance node. These substructures are explicitly generated and represented as sparse feature vectors.

* The Weisfeiler-Lehman (WL) graph kernel for RDF [17] counts full subtrees in the subgraph around the instance node. This kernel has two parameters, the subgraph depth $d$ and the number of iterations $h$ (which determines the depth of the subtrees). Following the settings in [15], we use two pairs of settings, $d=2, h=2$ (WL_2_2) and $d=4, h=3$ (WL_4_3).

* The Intersection Tree Path kernel for RDF [17] counts the walks in the subtree that spans from the instance node. Only the walks that go through the instance node are considered. We will therefore refer to it as the root Walk Count (WC) kernel. The root WC kernel has one parameter: the length of the paths $l$, for which we test 4 (WC_4) and 6 (WC_6), following the settings in [15].

Furthermore, we compare the results to the state-ofthe art graph embeddings approaches: TransE, TransH and TransR. These approaches have shown comparable results with the rest of the graph embeddings approaches on the task of link predictions. But most importantly, while there are many graph embeddings approaches, like RESCAL [53], Neural Tensor Networks (NTN) [85], ComplEx [89], HolE [52] and others, the approaches based on translating embeddings approaches scale to large knowledge-graphs as DBpedia. ${ }^{9}$ We use an existing implementation and build models on the small RDF datasets and the whole DBpedia data with the default parameters. ${ }^{10}$ For all the models we train 1,000 epochs and build vectors with size 100 . We have to note that the primary goal of such embeddings is the link prediction task, not standard machine learning tasks.

We perform no feature selection in any of the experiments, i.e., we use all the features generated with the given feature generation strategy. For the baseline feature generation strategies, we use binary feature vec-

\footnotetext{
${ }^{9}$ Because of high processing requirements we were not able to build the models for the Wikidata dataset.

${ }^{10}$ https://github.com/thunlp/KB2E/
} 
Table 2

3

4

5

6

7

9

10

\begin{tabular}{|c|c|c|c|c|c|c|c|c|c|c|c|c|c|c|c|c|}
\hline \multirow[t]{2}{*}{ Strategy/Dataset } & \multicolumn{4}{|c|}{ AIFB } & \multicolumn{4}{|c|}{ MUTAG } & \multicolumn{4}{|c|}{ BGS } & \multicolumn{4}{|c|}{$\mathrm{AM}$} \\
\hline & NB & KNN & SVM & $\mathrm{C} 4.5$ & NB & KNN & SVM & $\mathrm{C} 4.5$ & NB & KNN & SVM & $\mathrm{C} 4.5$ & NB & KNN & SVM & $\mathrm{C} 4.5$ \\
\hline rel in & 16.99 & 47.19 & 50.70 & 50.62 & 1 & 1 & 1 & 1 & 61.76 & 54.67 & 63.76 & 63.76 & 12.0 & 36.62 & 37.54 & 37.37 \\
\hline rel out & 45.07 & 45.56 & 50.70 & 51.76 & 41.18 & 54.41 & 62.94 & 62.06 & 54.76 & 69.05 & 72.70 & 69.33 & 18.57 & 63.46 & 63.84 & 63.6 \\
\hline rel in \& out & 25.59 & 51.24 & 50.80 & 51.80 & 1 & 1 & 1 & 1 & 54.76 & 67.00 & 72.00 & 70.00 & 18.42 & 63.00 & 64.38 & 63.96 \\
\hline rel-vals in & 73.24 & 54.54 & 81.86 & 80.75 & 1 & 1 & 1 & 1 & 79.48 & 83.52 & 86.50 & 68.57 & 13.36 & 38.55 & 41.74 & 34.72 \\
\hline rel-vals out & 86.86 & 55.69 & 82.39 & 71.73 & 62.35 & 62.06 & 73.53 & 62.94 & 84.95 & 65.29 & 83.10 & 73.38 & 82.17 & 53.17 & 85.75 & 84.6 \\
\hline rel-vals in\&out & 87.42 & 57.91 & 88.57 & 85.82 & 1 & 1 & 1 & 1 & 84.95 & 70.81 & 85.80 & 72.67 & 82.17 & 52.56 & 86.55 & 86. \\
\hline$N L \_2 \_2$ & 85.69 & 53.30 & 92.68 & 71.08 & 91.12 & 62.06 & 92.59 & 93.29 & 85.48 & 63.62 & 82.14 & 75.29 & 83.80 & 63.49 & 87.97 & 87.1 \\
\hline WL_4_3 & 85.65 & 65.95 & 83.43 & 89.25 & 70.59 & 62.06 & 94.29 & 93.47 & 90.33 & 85.57 & 91.05 & 87.67 & 87.00 & 54.65 & 86.42 & 87.3 \\
\hline WC_4 & 86.24 & 60.27 & 75.03 & 71.05 & 90.94 & 62.06 & 91.76 & 93.82 & 84.81 & 69.00 & 83.57 & 76.90 & 82.81 & 70.43 & 87.73 & 88.12 \\
\hline WC_6 & 86.83 & 64.18 & 82.97 & 71.05 & 92.00 & 72.56 & 86.47 & 93.82 & 85.00 & 67.00 & 78.71 & 76.90 & 81.50 & 67.50 & 86.97 & 87.2 \\
\hline TransE & 82.29 & 90.85 & 89.74 & 62.94 & 72.65 & 47.65 & 72.65 & 65.29 & 61.52 & 70.67 & 65.71 & 63.57 & 56.66 & 71.23 & 75.48 & 75.0 \\
\hline TransH & 80.65 & 88.10 & 84.67 & 59.74 & 70.59 & 43.24 & 70.29 & 57.06 & 58.81 & 69.95 & 69.38 & 58.95 & 58.43 & 71.24 & 76.69 & 76. \\
\hline TransR & 80.03 & 90.26 & 89.74 & 58.53 & 72.35 & 46.76 & 72.94 & 59.12 & 61.33 & 61.76 & 64.43 & 57.48 & 52.45 & 73.40 & 77.40 & 76. \\
\hline W2V CBOW 200 & 70.00 & 69.97 & 79.48 & 65.33 & 74.71 & 72.35 & 80.29 & 74.41 & 56.14 & 74.00 & 74.71 & 67.38 & 74.95 & 76.67 & 81.76 & 79. \\
\hline W2V CBOW 500 & 69.97 & 69.44 & 82.88 & 73.40 & 75.59 & 70.59 & 82.06 & 72.06 & 55.43 & 73.95 & 74.05 & 65.86 & 82.48 & 83.84 & 86.67 & 77.17 \\
\hline W2V SG 200 & 76.76 & 71.67 & 87.39 & 65.36 & 70.00 & 71.76 & 77.94 & 68.53 & 66.95 & 69.10 & 75.29 & 71.24 & 81.17 & 82.18 & 87.23 & 83.7 \\
\hline W2V SG 500 & 76.67 & 76.18 & 89.55 & 71.05 & 72.35 & 72.65 & 78.24 & 68.24 & 68.38 & 71.19 & 78.10 & 63.00 & 82.48 & 81.43 & 88.21 & 86.2 \\
\hline K2V CBOW 200 & 85.16 & 84.48 & 87.48 & 76.08 & 78.82 & 69.41 & 86.47 & 68.53 & 93.14 & 95.57 & 94.71 & 88.19 & 80.35 & 81.74 & 86.37 & 82. \\
\hline K2V CBOW 500 & 90.98 & 88.17 & 86.83 & 76.18 & 80.59 & 70.88 & 90.88 & 66.76 & 93.48 & 95.67 & 94.82 & 87.26 & 80.01 & 80.55 & 86.09 & 81. \\
\hline K2V SG 200 & 85.65 & 87.96 & 90.82 & 75.26 & 78.53 & 69.29 & 95.88 & 66.00 & 91.19 & 93.24 & 95.95 & 87.05 & 83.04 & 81.87 & 88.35 & 87.2 \\
\hline K2V SG 500 & 88.73 & 88.66 & 93.41 & 69.90 & 82.06 & 70.29 & 96.18 & 66.18 & 91.81 & 93.19 & 96.33 & 80.76 & 83.32 & 82.12 & 88.83 & 86. \\
\hline
\end{tabular}

tors, i.e., 1 if the feature exists for the instance, 0 otherwise.

We perform two learning tasks, i.e., classification and regression. For classification tasks, we use Naive Bayes, k-Nearest Neighbors $(k=3)$, C4.5 decision tree, and Support Vector Machines (SVMs). For the SVM classifier we optimize the complexity constant $C^{11}$ in the range $\left\{10^{-3}, 10^{-2}, 0.1,1,10,10^{2}, 10^{3}\right\}$. For regression, we use Linear Regression, M5Rules, and $\mathrm{k}$-Nearest Neighbors $(k=3)$. We measure accuracy for classification tasks, and root mean squared error (RMSE) for regression tasks. The results are calculated using stratified 10-fold cross validation.

The strategies for creating propositional features from Linked Open Data are implemented in the RapidMiner LOD extension ${ }^{12}[66,72]$. The experiments, including the feature generation and the evaluation, were

\footnotetext{
${ }^{11}$ The complexity constant sets the tolerance for misclassification, where higher C values allow for "softer" boundaries and lower values create "harder" boundaries.

${ }^{12} \mathrm{http} / / / d w s . i n f o r m a t i k . u n i-m a n n h e i m . d e / e n / r e s e a r c h /$ rapidminer-lod-extension
}

performed using the RapidMiner data analytics platform. ${ }^{13}$ The RapidMiner processes and the complete results can be found online. ${ }^{14}$ The experiments were run using a Linux machine with 20 GB RAM and 4 Intel Xeon 2.60 GHz CPUs.

\subsection{Results}

The results for the task of classification on the small RDF datasets are shown in Table 2. ${ }^{15}$ Experiments marked with "I" did not finish within ten days, or have run out of memory. The reason for that is the high number of generated features for some of the strategies, as explained in Section 5.4. From the results, we can observe that the $\mathrm{K} 2 \mathrm{~V}$ approach outperforms all the other approaches. More precisely, using the skip-gram fea-

\footnotetext{
$13 \mathrm{https}: / /$ rapidminer.com/

${ }^{14}$ http://data.dws.informatik.uni-mannheim.de/rmlod/LOD_ML_ Datasets/

${ }^{15}$ We do not consider the strategies for features derived from specific relations, i.e., types and categories, because the datasets do not contain categories, and all the instances are of the same type.
} 
ture vectors of size 500 in an SVM model provides the best results on all three datasets. The W2V approach on all three datasets performs closely to the standard graph substructure feature generation strategies, but it does not outperform them. K2V outperforms W2V because it is able to capture more complex substructures in the graph, like sub-trees, while W2V focuses only on graph paths. Furthermore, the related approaches, perform rather well on the AIFB dataset, and achieve comparable results to the $\mathrm{K} 2 \mathrm{~V}$ approach, however, on the other two dataset $\mathrm{K} 2 \mathrm{~V}$ significantly outperforms all three of them.

The results for the task of classification and regression on the five datasets using the DBpedia and Wikidata entities' vectors are shown in Tables 3 and 4 . We can observe that the latent vectors extracted from DBpedia and Wikidata outperform all of the standard feature generation approaches. Furthermore, the RDF2vec approaches built on the DBpedia dataset continuously outperform the related approaches, i.e., TransE, TransH, and TransR, on both tasks for all the datasets, except on the Forbes dataset for the task of classification. In this case, all the related approaches outperform the baseline approaches as well as the RDF2vec approach. The difference is most significant when using the $\mathrm{C} 4.5$ classifier. In general, the DBpedia vectors work better than the Wikidata vectors, where the skip-gram vectors with size 200 or 500 built on graph walks of depth 8 on most of the datasets lead to the best performance. An exception is the AAUP dataset, where the Wikidata skip-gram 500 vectors outperform the other approaches. Furthermore, in Table we can notice that the SVM model achieves very low accuracy on the AAUP dataset. The explanation for such poor performance might be that the instances in the dataset are not linearly separable, therefore the SVM is unable to correctly classify the instances.

On both tasks, we can observe that the skip-gram vectors perform better than the CBOW vectors. Furthermore, the vectors with higher dimensionality and longer walks lead to a better representation of the entities and better performance on most of the datasets. However, for the variety of tasks at hand, there is no universal approach, i.e., a combination of an embedding model and a machine learning method, that consistently outperforms the others.

The structure and quality of the underlying knowledge graph may influence the results of the algorithms. For example, there are quite a few differences in the average degree of resources in different knowledge graphs [71], which influence the walks and ultimately the embeddings. For the datasets at hand, the entities are cities, movies, albums, universities, and companies. Table 5 shows the average outgoing degrees for the entities in those classes, in the respective knowledge graphs. This means that for most of the cases, the entities at hand are, on average, described at more detail in DBpedia, i.e., we can expect that also the embeddings for DBpedia will be of higher quality. It is further remarkable that the biggest discrepancy for the classification task between the best DBpedia and the best Wikidata embeddings occurs for the Metacritic albums dataset (with 15 percentage points), which is also the class with the highest difference in the degree distribution. On the other hand, for universities, which have a high level of detail in both knowledge graphs, the difference is not that severe (and Wikidata embeddings are even superior on the regression task). However, the number of datasets is still too small to make a quantitative statement about the relation between level of detail in the knowledge graph and performance of the embeddings. Similar observation holds in the entity and document modeling evaluation (see Section 6), and the recommender systems evaluation (see Section 7).

\subsection{Semantics of vector representations}

To analyze the semantics of the vector representations, we employ Principal Component Analysis (PCA) to project the entities' feature vectors into a two dimensional feature space. We selected seven countries and their capital cities, and visualized their vectors as points in a two-dimensional space. Figure 2(a) shows the corresponding DBpedia vectors, and Fig. 2(b) shows the corresponding Wikidata vectors. The figure illustrates the ability of the model to automatically organize entities of different types, and preserve the relationships between different entities. For example, we can see that there is a clear separation between the countries and the cities, and the relation "capital" between each pair of country and the corresponding capital city is preserved. Furthermore, we can observe that more similar entities are positioned closer to each other, e.g., we can see that the countries that are part of the EU are closer to each other, and the same applies for the Asian countries.

\subsection{Features increase rate}

Finally, we conduct a scalability experiment, where we examine how the number of instances affects the 
Table 3

Classification results. The first number represents the dimensionality of the vectors, while the second number represent the value for the depth parameter. The best results are marked in bold. Experiments marked with "”" did not finish within ten days, or have run out of memory

\begin{tabular}{|c|c|c|c|c|c|c|c|c|c|c|c|c|c|c|c|c|c|c|c|c|}
\hline \multirow[t]{2}{*}{ Strategy/Dataset } & \multirow{2}{*}{$\mathrm{NB}$} & \multicolumn{3}{|c|}{ Cities } & \multicolumn{4}{|c|}{ Metacritic Movies } & \multicolumn{4}{|c|}{ Metacritic Albums } & \multicolumn{4}{|c|}{ AAUP } & \multicolumn{4}{|c|}{ Forbes } \\
\hline & & KNN & SVM & $\mathrm{C} 4.5$ & NB & KNN & SVM & C4.5 & NB & KNN & SVM & C 4.5 & NB & KNN & SVM & C4.5 & NB & KNN & SVM & C4.5 \\
\hline pes & 55.71 & 56.17 & 63.21 & 59.05 & 68.00 & 57.60 & 71.40 & 70.00 & 66.50 & 50.75 & 62.31 & 54.44 & 41.00 & 85.62 & 91.67 & 92.78 & 55.08 & 75.84 & 75.67 & 75.85 \\
\hline categories & .74 & 49.98 & .39 & 56.17 & 75.25 & 62.70 & 76.35 & 69.50 & 67.40 & 54.13 & 64.50 & 56.62 & 48.00 & 85.83 & 90.78 & 91.87 & 50.38 & 76.11 & 75.70 & 75.70 \\
\hline rel in & 60.41 & 58.46 & 71.70 & 60.35 & 52.75 & 49.90 & 60.35 & 60.10 & 51.13 & 62.19 & 65.25 & 60.75 & 45.63 & 85.94 & 90.62 & 92.81 & 50.24 & 76.49 & 75.16 & 76.10 \\
\hline rel out & 47.62 & 60.00 & 66.04 & 56.71 & 52.90 & 58.45 & 66.40 & 62.70 & 58.75 & 63.75 & 62.25 & 64.50 & 41.15 & 85.83 & 89.58 & 91.35 & 64.73 & 75.84 & 75.73 & 75.92 \\
\hline rel in \& out & 59.44 & 58.57 & 6.04 & 56.47 & 52.95 & 59.30 & 67.75 & 62.55 & 58.69 & 64.50 & 67.38 & 61.56 & 42.71 & 85.94 & 89.67 & 92.50 & 22.27 & 75.96 & 76.34 & 75.98 \\
\hline rel-vals in & 1 & 1 & 1 & 1 & 50.60 & 50.00 & 50.60 & 50.00 & 50.88 & 50.00 & 50.81 & 50.00 & 54.06 & 84.69 & 89.51 & 1 & 14.95 & 76.15 & 76.97 & 75.73 \\
\hline rel-vals out & 53.79 & 35.91 & 55.66 & 64.13 & 78.50 & 54.78 & 78.71 & 1 & 74.06 & 52.56 & 76.99 & 1 & 57.81 & 85.73 & 91.46 & 91.78 & 67.09 & 75.61 & 75.74 & 76.74 \\
\hline rel-vals in\&o & 1 & 1 & 1 & 1 & 77.90 & 55.75 & 77.82 & 1 & 4.25 & 1.25 & 5.85 & 1 & 63.44 & 84.69 & 91.56 & 1 & 67.20 & 75.88 & 75.96 & 76.75 \\
\hline WL_2_2 & 70.98 & 49.31 & 65.34 & 75.29 & 75.45 & 66.90 & 79.30 & 70.80 & 73.63 & 64.69 & 76.25 & 62.00 & 58.33 & 91.04 & 91.46 & 92.40 & 64.17 & 75.71 & 75.10 & 76.59 \\
\hline WL_4_3 & 65.48 & 53.29 & 69.90 & 69.31 & 1 & & & 1 & 1 & 1 & 1 & 1 & 1 & 1 & 1 & 1 & 1 & 1 & 1 & 1 \\
\hline WC_4 & 72.71 & 47.39 & 66.48 & 75.13 & 75.39 & 65.89 & 74.93 & 69.08 & 72.00 & 60.63 & 76.88 & 63.69 & 57.29 & 90.63 & 93.44 & 92.60 & 64.23 & 75.77 & 76.22 & 76.47 \\
\hline WC_6 & 65.52 & 52.36 & 67.95 & 65.15 & 74.25 & 55.30 & 78.40 & & 72.81 & 52.87 & 77.94 & 1 & 57.19 & 90.73 & 90.94 & 92.60 & 64.04 & 75.65 & 76.22 & 76.59 \\
\hline DB_TransE & 65.79 & 75.71 & 74.63 & 61.50 & 65.75 & 64.17 & 68.96 & 61.16 & 62.81 & 60.48 & 64.17 & 56.86 & 80.28 & 84.86 & 28.95 & 89.65 & 92.88 & 79.98 & 74.37 & 95.44 \\
\hline DB_TransH & 64.39 & 72.66 & 76.66 & 60.89 & 63.51 & 63.25 & 67.43 & 60.96 & 63.97 & 63.13 & 65.07 & 60.23 & 80.39 & 84.86 & 27.55 & 89.21 & 93.82 & 79.98 & 74.37 & 93.68 \\
\hline DB_TransR & 63.08 & 67.32 & 74.50 & 59.84 & 64.38 & 60.16 & 64.43 & 52.04 & 63.56 & 59.68 & 66.41 & 60.39 & 79.19 & 84.86 & 28.95 & 89.00 & 93.28 & 79.98 & 74.37 & 93.70 \\
\hline DB2vec SG 200w 200v 4d & 75.66 & 73.50 & 75.68 & 47.58 & 77.58 & 78.24 & 79.61 & 72.78 & 73.85 & 73.79 & 75.28 & 65.95 & 78.21 & 85.18 & 29.57 & 91.83 & 82.24 & 81.07 & 74.10 & 85.23 \\
\hline DB2vec CBOW & 68.45 & 68.39 & 71.53 & 60.34 & 70.64 & 72.83 & 75.43 & 69.11 & 70.03 & 1 & 73.53 & 60.84 & 73.09 & 85.29 & 29.57 & 91.40 & 87.34 & 80.38 & 74.10 & 84.40 \\
\hline $\mathrm{B} 2 \mathrm{vec} \mathrm{CB}$ & 59.32 & 3.84 & 77.39 & 64.32 & 65.60 & 79.74 & 82.90 & 74.33 & 70.72 & 71.86 & 76.36 & 67.24 & 73.36 & 89.65 & 29.00 & 92.45 & 89.38 & 80.94 & 76.83 & 84. \\
\hline DB2vec CBOW 500w 500v 4d & 59.32 & 71.34 & 76.37 & 66.34 & 65.65 & 79.49 & 82.75 & 73.87 & 69.71 & 71.93 & 75.41 & 65.65 & 72.71 & 89.65 & 29.11 & 92.01 & 89.02 & 80.82 & 76.95 & 85.17 \\
\hline DB2vec SG 500w 200v 4d & 60.34 & 71.82 & 76.37 & 65.37 & 65.25 & 80.44 & 83.25 & 73.87 & 68.95 & 73.89 & 76.11 & 67.87 & 71.20 & 89.65 & 28.90 & 92.12 & 88.78 & 80.82 & 77.92 & 85.77 \\
\hline DB2vec SG 500w 500v 4d & 58.34 & 72.84 & 76.87 & 67.84 & 65.45 & 80.14 & 83.65 & 72.82 & 70.41 & 74.34 & 78.44 & 67.49 & 71.19 & 89.65 & 28.90 & 92.23 & 88.30 & 80.94 & 77.25 & 84.81 \\
\hline DB2vec CBOW 500w 200v 8d & 69.26 & 69.87 & 67.32 & 63.13 & 57.83 & 70.08 & 65.25 & 67.47 & 67.91 & 64.44 & 72.42 & 65,39 & 68.18 & 85.33 & 28.90 & 90.50 & 77.35 & 80.34 & 28.90 & 85.17 \\
\hline DB2vec CBO & 62.26 & 69.87 & 76.84 & 63.21 & 58.78 & 69.82 & 69.46 & 67.67 & 67.53 & 65.83 & 74.26 & 63.42 & 62.90 & 85.22 & 29.11 & 90.61 & 89.86 & 80.34 & 78.65 & 84.81 \\
\hline DB2vec SG 500w 200v 8d & 73.32 & 75.89 & 78.92 & 60.74 & 79.94 & 79.49 & 83.30 & 75.13 & 77.25 & 76.87 & 79.72 & 69.14 & 78.53 & 85.12 & 29.22 & 91.04 & 90.10 & 80.58 & 78.96 & 84.68 \\
\hline DB2vec SG 500w 500v 8d & 89.73 & 69.16 & 84.19 & 72.25 & 80.24 & 78.68 & 82.80 & 72.42 & 73.57 & 76.30 & 78.20 & 68.70 & 75.07 & 94.48 & 29.11 & 94.15 & 88.53 & 80.58 & 77.79 & 86.38 \\
\hline WD2vec CBOW 200w 200v 4d & 68.76 & 57.71 & 75.56 & 61.37 & 51.49 & 52.20 & 51.64 & 49.01 & 50.86 & 50.29 & 51.44 & 50.09 & 50.54 & 90.18 & 89.63 & 88.83 & 49.84 & 81.08 & 76.77 & 79.14 \\
\hline WD2vec CBOW 200w 500v 4d & 68.24 & 57.75 & 85.56 & 64.54 & 49.22 & 48.56 & 51.04 & 50.98 & 53.08 & 50.03 & 52.33 & 53.28 & 48.45 & 90.39 & 89.74 & 88.31 & 51.95 & 80.74 & 78.18 & 80.32 \\
\hline WD2vec SG 200w 200v 4d & 72.58 & 57.53 & 75.48 & 52.32 & 69.53 & 70.14 & 75.39 & 67.00 & 60.32 & 62.03 & 64.76 & 58.54 & 60.87 & 90.50 & 89.63 & 89.98 & 65.45 & 81.17 & 77.74 & 77.03 \\
\hline WD2vec SG 200w 500v 4d & 83.20 & 60.72 & 79.87 & 61.67 & 71.10 & 70.19 & 76.30 & 67.31 & 55.31 & 58.92 & 63.42 & 56.63 & 55.85 & 90.60 & 89.63 & 87.69 & 58.95 & 81.17 & 79.00 & 79.56 \\
\hline
\end{tabular}




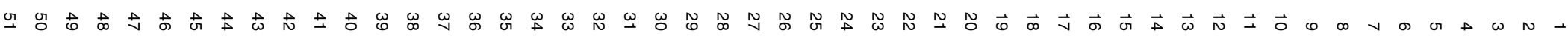

Table 4

Regression results. The first number represents the dimensionality of the vectors, while the second number represent the value for the depth parameter. The best results are marked in bold. Experiments that did not finish within ten days, or that have run out of memory are marked with ""

\begin{tabular}{|c|c|c|c|c|c|c|c|c|c|c|c|c|c|c|c|}
\hline \multirow[t]{2}{*}{ Strategy/Dataset } & \multicolumn{3}{|c|}{ Cities } & \multicolumn{3}{|c|}{ Metacritic Movies } & \multicolumn{3}{|c|}{ Metacritic Albums } & \multicolumn{3}{|c|}{ AAUP } & \multicolumn{3}{|c|}{ Forbes } \\
\hline & LR & KNN & M5 & LR & KNN & M5 & LR & KNN & M5 & LR & $\mathrm{KNN}$ & M5 & LR & KNN & M5 \\
\hline types & 24.30 & 22.16 & 18.79 & 77.80 & 30.68 & 22.16 & 16.45 & 18.36 & 13.95 & 9.83 & 34.95 & 6.28 & 29.22 & 21.07 & 18.32 \\
\hline categories & 18.88 & 22.68 & 22.32 & 84.57 & 23.87 & 22.50 & 16.73 & 16.64 & 13.95 & 8.08 & 34.94 & 6.16 & 19.16 & 21.48 & 18.39 \\
\hline rel in & 49.87 & 18.53 & 19.21 & 22.60 & 41.40 & 22.56 & 13.50 & 22.06 & 13.43 & 9.69 & 34.98 & 6.56 & 27.56 & 20.93 & 18.60 \\
\hline rel out & 49.87 & 18.53 & 19.21 & 21.45 & 24.42 & 20.74 & 13.32 & 14.59 & 13.06 & 8.82 & 34.95 & 6.32 & 21.73 & 21.11 & 18.97 \\
\hline rel in \& out & 40.80 & 18.21 & 18.80 & 21.45 & 24.42 & 20.74 & 13.33 & 14.52 & 12.91 & 12.97 & 34.95 & 6.36 & 26.44 & 20.98 & 19.54 \\
\hline rel-vals in & 1 & 1 & 1 & 21.46 & 24.19 & 20.43 & 13.94 & 23.05 & 13.95 & 1 & 34.96 & 6.27 & 1 & 20.86 & 19.31 \\
\hline rel-vals out & 20.93 & 23.87 & 20.97 & 25.99 & 32.18 & 22.93 & 1 & 15.28 & 13.34 & 1 & 34.95 & 6.18 & 1 & 20.48 & 18.37 \\
\hline rel-vals in\&out & 1 & 1 & 1 & 1 & 25.37 & 20.96 & 1 & 15.47 & 13.33 & 1 & 34.94 & 6.18 & 1 & 20.20 & 18.20 \\
\hline WL_2_2 & 20.21 & 24.60 & 20.85 & 1 & 21.62 & 19.84 & 1 & 13.99 & 12.81 & 1 & 34.96 & 6.27 & 1 & 19.81 & 19.49 \\
\hline WL_4_3 & 17.79 & 20.42 & 17.04 & 1 & 1 & 1 & 1 & 1 & 1 & 1 & 1 & 1 & 1 & 1 & 1 \\
\hline WC_4 & 20.33 & 25.95 & 19.55 & 1 & 22.80 & 22.99 & 1 & 14.54 & 12.87 & 9.12 & 34.95 & 6.24 & 1 & 20.45 & 19.26 \\
\hline WC_6 & 19.51 & 33.16 & 19.05 & 1 & 23.86 & 19.19 & 1 & 19.51 & 13.02 & 1 & 35.39 & 6.31 & 1 & 20.58 & 19.04 \\
\hline DB_TransE & 14.22 & 14.45 & 14.46 & 20.66 & 23.61 & 20.71 & 13.20 & 14.71 & 13.23 & 6.34 & 57.27 & 6.43 & 20.00 & 21.55 & 17.73 \\
\hline DB_TransH & 13.88 & 12.81 & 14.28 & 20.71 & 23.59 & 20.72 & 13.04 & 14.19 & 13.03 & 6.35 & 57.27 & 6.47 & 19.88 & 21.54 & 16.66 \\
\hline DB_TransR & 14.50 & 13.24 & 14.57 & 20.10 & 23.37 & 20.04 & 13.87 & 15.74 & 13.93 & 6.34 & 57.31 & 6.37 & 20.45 & 21.55 & 17.18 \\
\hline DB2vec SG 200w 200v 4d & 14.26 & 14.02 & 15.15 & 16.82 & 18.52 & 16.56 & 11.57 & 12.38 & 11.56 & 6.26 & 57.20 & 6.45 & 19.68 & 20.85 & 18.50 \\
\hline DB2vec CBOW 200w 200v 4d & 14.13 & 13.33 & 15.46 & 18.66 & 20.61 & 18.39 & 12.32 & 13.87 & 12.32 & 6.35 & 56.85 & 6.37 & 19.67 & 20.84 & 18.72 \\
\hline DB2vec CBOW 500w 200v 4d & 14.37 & 12.55 & 14.33 & 15.90 & 17.46 & 15.89 & 11.79 & 12.45 & 11.59 & 12.13 & 45.76 & 12.00 & 18.32 & 26.19 & 17.43 \\
\hline DB2vec CBOW 500w 500v 4d & 14.99 & 12.46 & 14.66 & 15.90 & 17.45 & 15.73 & 11.49 & 12.60 & 11.48 & 12.44 & 45.67 & 12.30 & 18.23 & 26.27 & 17.62 \\
\hline DB2vec SG 500w 200v 4d & 13.38 & 12.54 & 15.13 & 15.81 & 17.07 & 15.84 & 11.30 & 12.36 & 11.42 & 12.13 & 45.72 & 12.10 & 17.63 & 26.13 & 17.85 \\
\hline DB2vec SG 500w 500v 4d & 14.73 & 13.25 & 16.80 & 15.66 & 17.14 & 15.67 & 11.20 & 12.11 & 11.28 & 12.09 & 45.76 & 11.93 & 18.23 & 26.09 & 17.74 \\
\hline DB2vec CBOW 500w 200v 8d & 16.17 & 17.14 & 17.56 & 21.55 & 23.75 & 21.46 & 13.35 & 15.41 & 13.43 & 6.47 & 55.76 & 6.47 & 24.17 & 26.48 & 22.61 \\
\hline DB2vec CBOW 500w 500v 8d & 18.13 & 17.19 & 18.50 & 20.77 & 23.67 & 20.69 & 13.20 & 15.14 & 13.25 & 6.54 & 55.33 & 6.55 & 21.16 & 25.90 & 20.33 \\
\hline DB2vec SG 500w 200v 8d & 12.85 & 14.95 & 12.92 & 15.15 & 17.13 & 15.12 & 10.90 & 11.43 & 10.90 & 6.22 & 56.95 & 6.25 & 18.66 & 21.20 & 18.57 \\
\hline DB2vec SG 500w 500v 8d & 11.92 & 12.67 & 10.19 & 15.45 & 17.80 & 15.50 & 10.89 & 11.72 & 10.97 & 6.26 & 56.95 & 6.29 & 18.35 & 21.04 & 16.61 \\
\hline WD2vec CBOW 200w 200v 4d & 20.15 & 17.52 & 20.02 & 23.54 & 25.90 & 23.39 & 14.73 & 16.12 & 14.55 & 16.80 & 42.61 & 6.60 & 27.48 & 22.60 & 21.77 \\
\hline WD2vec CBOW 200w 500v 4d & 23.76 & 18.33 & 20.39 & 24.14 & 22.18 & 24.56 & 14.09 & 16.09 & 14.00 & 13.08 & 42.89 & 6.08 & 50.23 & 21.92 & 26.66 \\
\hline WD2vec SG 200w 200v 4d & 20.47 & 18.69 & 20.72 & 19.72 & 21.44 & 19.10 & 13.51 & 13.91 & 13.67 & 6.86 & 42.82 & 6.52 & 23.69 & 21.59 & 20.49 \\
\hline WD2vec SG 200w 500v 4d & 22.25 & 19.41 & 19.23 & 25.99 & 21.26 & 19.19 & 13.23 & 14.96 & 13.25 & 8.27 & 42.84 & 6.05 & 21.98 & 21.73 & 21.58 \\
\hline
\end{tabular}


Table 5

Average outgoing degree for selected classes

\begin{tabular}{lcc}
\hline Class & DBpedia & Wikidata \\
\hline City & 129.74 & 84.47 \\
Movie & 64.28 & 57.08 \\
Album & 64.13 & 22.86 \\
University & 60.01 & 34.55 \\
Company & 50.40 & 19.03 \\
Book & 45.70 & 23.54 \\
Music Artist & 80.86 & 34.19 \\
\hline
\end{tabular}
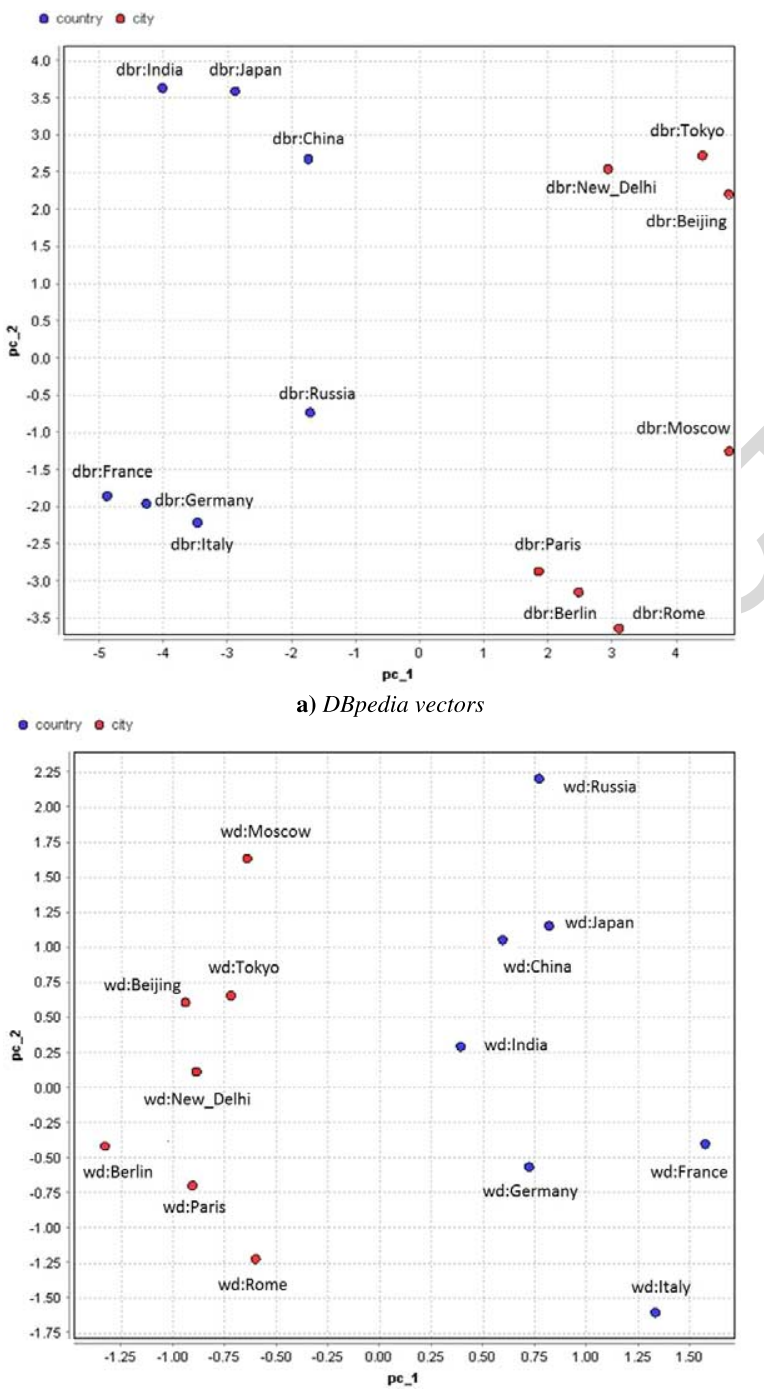

b) Wikidata vectors

Fig. 2. Two-dimensional PCA projection of the 500-dimensional Skip-gram vectors of countries and their capital cities. number of generated features by each feature generation strategy. For this purpose we use the Metacritic Movies dataset. We start with a random sample of 100 instances, and in each next step we add 200 (or 300) unused instances, until the complete dataset is used, i.e., 2,000 instances. The number of generated features for each sub-sample of the dataset using each of the feature generation strategies is shown in Fig. 3.

From the chart, we can observe that the number of generated features sharply increases when adding more samples in the datasets, especially for the strategies based on graph substructures.

In contrast, the number of features remains constant when using the RDF2Vec approach, as it is fixed to 200 or 500, respectively, independently of the number of samples in the data. Thus, by design, it scales to larger datasets without increasing the dimensionality of the dataset.

\section{Entity and document modeling}

Calculating entity relatedness and similarity are fundamental problems in numerous tasks in information retrieval, natural language processing, and Web-based knowledge extraction. While similarity only considers subsumption relations to assess how two objects are alike, relatedness takes into account a broader range of relations, i.e., the notion of relatedness is wider than that of similarity. For example, "Facebook" and "Google" are both entities of the class company, and they have high similarity and relatedness score. On the other hand, "Facebook" and "Mark Zuckerberg" are not similar at all, but are highly related, while "Google" and "Mark Zuckerberg" are not similar at all, and have lower relatedness value compared to the first pair of entities.

\subsection{Approach}

In this section, we introduce several approaches for entity and document modeling based on the previously built latent feature vectors for entities.

\subsubsection{Entity similarity}

As previously mentioned, in the RDF2 Vec feature embedding space (see Section 3), semantically similar entities appear close to each other in the feature space. Therefore, the problem of calculating the similarity between two instances is a matter of calculating the distance between two instances in the given 


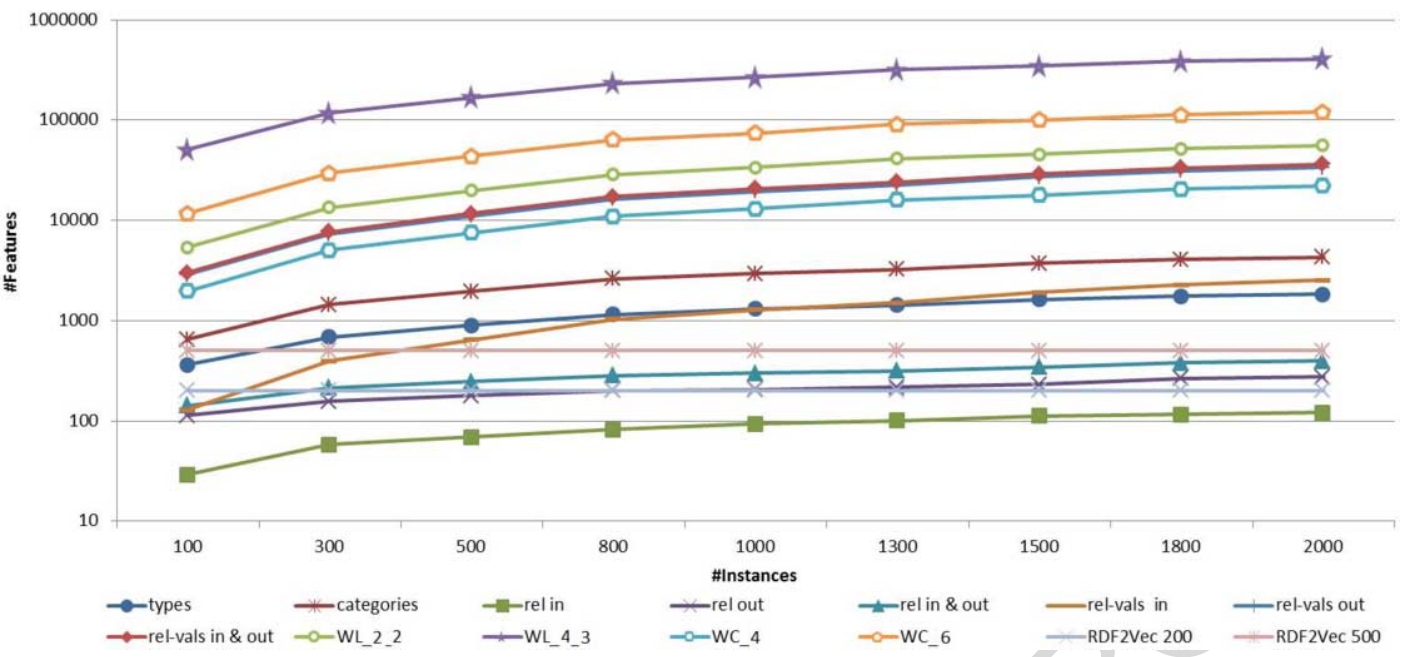

Fig. 3. Features increase rate per strategy (log scale).

feature space. To do so, we use the standard cosine similarity measure, which is applied on the vectors of the entities. Formally, the similarity between two entities $e_{1}$ and $e_{2}$, with vectors $V_{1}$ and $V_{2}$, is calculated as the cosine similarity between the vectors $V_{1}$ and $V_{2}$ :

$$
\operatorname{sim}\left(e_{1}, e_{2}\right)=\frac{V_{1} \cdot V_{2}}{\left\|V_{1}\right\| \cdot\left\|V_{2}\right\|}
$$

\subsubsection{Document similarity}

We use those entity similarity scores in the task of calculating semantic document similarity. We follow a similar approach as the one presented in [62], where two documents are considered to be similar if many entities of the one document are similar to at least one entity in the other document. More precisely, we try to identify the most similar pairs of entities in both documents, ignoring the similarity of all other pairs.

Given two documents $d_{1}$ and $d_{2}$, the similarity between the documents $\operatorname{sim}\left(d_{1}, d_{2}\right)$ is calculated as follows:

1. Extract the sets of entities $E_{1}$ and $E_{2}$ in the documents $d_{1}$ and $d_{2}$.

2. Calculate the similarity score $\operatorname{sim}\left(e_{1 i}, e_{2 j}\right)$ for each pair of entities in document $d_{1}$ and $d_{2}$, where $e_{1 i} \in E_{1}$ and $e_{2 j} \in E_{2}$

3. For each entity $e_{1 i}$ in $d_{1}$ identify the maximum similarity to an entity in $d_{2} \max \_\operatorname{sim}\left(e_{1 i}, e_{2 j} \in\right.$ $\left.E_{2}\right)$, and vice versa.
4. Calculate the similarity score between the documents $d_{1}$ and $d_{2}$ as:

$$
\begin{aligned}
\operatorname{sim}( & \left.d_{1}, d_{2}\right) \\
= & \left(\sum_{i=1}^{\left|E_{1}\right|} \max _{-} \operatorname{sim}\left(e_{1 i}, e_{2 j} \in E_{2}\right)\right. \\
& \left.+\sum_{j=1}^{\left|E_{2}\right|} \max _{-} \operatorname{sim}\left(e_{2 j}, e_{1 i} \in E_{1}\right)\right) \\
& /\left(\left|E_{1}\right|+\left|E_{2}\right|\right)
\end{aligned}
$$

\subsubsection{Entity relatedness}

In this approach we assume that two entities are related if they often appear in the same context. For example, "Facebook" and "Mark Zuckerberg", which are highly related, are often used in the same context in many sentences. To calculate the probability of two entities being in the same context, we make use of the RDF2Vec models and the set of sequences of entities generated as described in Section 3. Given a RDF2vec model and a set of sequences of entities, we calculate the relatedness between two entities $e_{1}$ and $e_{2}$, as the probability $p\left(e_{1} \mid e_{2}\right)$ calculated using the softmax function. In the case of a CBOW model, the probability is calculated as:

$$
p\left(e_{1} \mid e_{2}\right)=\frac{\exp \left(v_{e_{2}}^{T} v_{e_{1}}^{\prime}\right)}{\sum_{e=1}^{V} \exp \left(v_{e_{2}}^{T} v_{e}^{\prime}\right)},
$$


Similarity-based relatedness Spearman's rank correlation results

\begin{tabular}{|c|c|c|c|c|c|c|}
\hline Model & IT companies & Hollywood Celebrities & Television Series & Video Games & Chuck Norris & All 21 entities \\
\hline DB2vec SG 200w 200v 4d & 0.525 & 0.505 & 0.532 & 0.571 & 0.439 & 0.529 \\
\hline DB2vec CBOW 200w 200v & 0.330 & 0.294 & 0.462 & 0.399 & 0.179 & 0.362 \\
\hline DB2vec CBOW 500w 200v 4d & 0.538 & 0.560 & 0.572 & 0.596 & 0.500 & 0.564 \\
\hline DB2vec CBOW 500w 500v 4d & 0.546 & 0.544 & 0.564 & 0.606 & 0.496 & 0.562 \\
\hline DB2vec SG 500w 200v 4d & 0.508 & 0.546 & 0.497 & 0.634 & 0.570 & 0.547 \\
\hline DB2vec SG 500w 500v 4d & 0.507 & 0.538 & 0.505 & 0.611 & 0.588 & 0.542 \\
\hline DB2vec CBOW 500w 200v 8d & 0.611 & 0.495 & 0.315 & 0.443 & 0.365 & 0.461 \\
\hline DB2vec CBOW 500w 500v 8w & 0.486 & 0.507 & 0.285 & 0.440 & 0.470 & 0.432 \\
\hline DB2vec SG 500w 200v 8w & 0.739 & 0.723 & 0.526 & 0.659 & 0.625 & 0.660 \\
\hline DB2vec SG 500w 500v 8w & 0.743 & 0.734 & 0.635 & 0.669 & 0.628 & 0.692 \\
\hline WD2vec CBOW 200w 200v 4d & 0.246 & 0.418 & 0.156 & 0.374 & 0.409 & 0.304 \\
\hline WD2vec CBOW 200w 500v 4d & 0.190 & 0.403 & 0.103 & 0.106 & 0.150 & 0.198 \\
\hline WD2vec SG 200w 200v 4d & 0.502 & 0.604 & 0.405 & 0.578 & 0.279 & 0.510 \\
\hline WD2vec SG 200w 500v 4d & 0.464 & 0.562 & 0.313 & 0.465 & 0.168 & 0.437 \\
\hline Wiki2vec & 0.613 & 0.544 & 0.334 & 0.618 & 0.436 & 0.523 \\
\hline
\end{tabular}

where $v_{e}^{\prime}$ is the output vector of the entity $e$, and $V$ is the complete vocabulary of entities.

In the case of a skip-gram model, the probability is calculated as:

$$
p\left(e_{1} \mid e_{2}\right)=\frac{\exp \left(v_{e_{1}}^{\prime T} v_{e_{2}}\right)}{\sum_{e=1}^{V} \exp \left(v_{e}^{\prime T} v_{e_{2}}\right)},
$$

where $v_{e}$ and $v_{e}^{\prime}$ are the input and the output vector of the entity $e$, and $V$ is the complete vocabulary of entities.

\subsection{Evaluation}

For both tasks of determining entity relatedness and document similarity, we use existing benchmark datasets to compare the use of RDF2Vec models against state of the art approaches.

\subsubsection{Entity relatedness}

For evaluating the entity relatedness approach, we use the KORE dataset [29]. The dataset consists of 21 main entities, whose relatedness to the other 20 entities each has been manually assessed, leading to 420 rated entity pairs. We use the Spearman's rank correlation as an evaluation metric.

We use two approaches for calculating the relatedness rank between the entities, i.e. (i) the entity similarity approach described in Section 6.1.1; (ii) the entity relatedness approach described in Section 6.1.3.

We evaluate each of the RDF2Vec models separately. Furthermore, we also compare to the Wiki2vec model, ${ }^{16}$ which is built on the complete Wikipedia corpus, and provides vectors for each DBpedia entity.

Table 6 shows the Spearman's rank correlation results when using the entity similarity approach. Table 7 shows the results for the relatedness approach. The results show that the DBpedia models outperform the Wikidata models. Increasing the number of walks per entity improves the results. Also, the skip-gram models outperform the CBOW models continuously. We can observe that the relatedness approach outperforms the similarity approach.

Furthermore, we compare our approaches to several state-of-the-art graph-based entity relatedness approaches:

- baseline: computes entity relatedness as a function of distance between the entities in the network, as described in [82].

- KORE: calculates keyphrase overlap relatedness, as described in the original KORE paper [29].

- CombIC: semantic similarity using a Graph Edit Distance based measure [82].

- ER: Exclusivity-based relatedness [32].

The comparison, shown in Table 8 , shows that our entity relatedness approach outperforms all the rest for each category of entities. Interestingly enough, the entity similarity approach, although addressing a different task, also outperforms the majority of state of the art approaches.

\footnotetext{
${ }^{16}$ https://github.com/idio/wiki2vec
} 
Table 7

Context-based relatedness Spearman's rank correlation results

\begin{tabular}{lcccccc}
\hline Model & IT companies & Hollywood Celebrities & Television Series & Video Games & Chuck Norris & All 21 entities \\
\hline DB2vec SG 200w 200v 4d & 0.643 & 0.547 & 0.583 & 0.428 & 0.591 & 0.552 \\
DB2vec CBOW 200w 200v & 0.361 & 0.326 & 0.467 & 0.426 & 0.208 & 0.386 \\
DB2vec CBOW 500w 200v 4d & 0.671 & 0.566 & 0.591 & 0.434 & 0.609 & 0.568 \\
DB2vec CBOW 500w 500v 4d & 0.672 & 0.622 & 0.578 & 0.440 & 0.581 & 0.578 \\
DB2vec SG 500w 200v 4d & 0.666 & 0.449 & 0.611 & 0.360 & 0.630 & 0.526 \\
DB2vec SG 500w 500v 4d & 0.667 & 0.444 & 0.609 & 0.389 & 0.668 & 0.534 \\
DB2vec CBOW 500w 200v 8d & 0.579 & 0.484 & 0.368 & 0.460 & 0.412 & 0.470 \\
DB2vec CBOW 500w 500v 8d & 0.552 & 0.522 & 0.302 & 0.487 & 0.665 & 0.475 \\
DB2vec SG 500w 200v 8d & $\mathbf{0 . 8 1 1}$ & $\mathbf{0 . 7 7 8}$ & $\mathbf{0 . 7 1 1}$ & $\mathbf{0 . 6 5 8}$ & $\mathbf{0 . 6 7 0}$ & $\mathbf{0 . 7 3 6}$ \\
DB2vec SG 500w 500v 8d & 0.748 & 0.729 & 0.689 & 0.537 & 0.625 & 0.673 \\
WD2vec CBOW 200w 200v 4d & 0.287 & 0.241 & -0.025 & 0.311 & 0.226 & 0.205 \\
WD2vec CBOW 200w 500v 4d & 0.166 & 0.215 & 0.233 & 0.335 & 0.344 & 0.243 \\
WD2vec SG 200w 200v 4d & 0.574 & 0.671 & 0.504 & 0.410 & 0.079 & 0.518 \\
WD2vec SG 200w 500v 4d & 0.661 & 0.639 & 0.537 & 0.395 & 0.474 & 0.554 \\
Wiki2vec & 0.291 & 0.296 & 0.406 & 0.353 & 0.175 & 0.329 \\
\hline
\end{tabular}

Table 8

Spearman's rank correlation results comparison to related work

\begin{tabular}{|c|c|c|c|c|c|c|}
\hline Approach & IT companies & Hollywood Celebrities & Television Series & Video Games & Chuck Norris & All 21 entities \\
\hline baseline & 0.559 & 0.639 & 0.529 & 0.451 & 0.458 & 0.541 \\
\hline KORE & 0.759 & 0.715 & 0.599 & 0.760 & 0.498 & 0.698 \\
\hline CombIC & 0.644 & 0.690 & 0.643 & 0.532 & 0.558 & 0.624 \\
\hline ER & 0.727 & 0.643 & 0.633 & 0.519 & 0.477 & 0.630 \\
\hline DB_TransE & -0.023 & 0.120 & -0.084 & 0.353 & -0.347 & 0.070 \\
\hline DB_TransH & -0.134 & 0.185 & -0.097 & 0.204 & -0.044 & 0.035 \\
\hline DB_TransR & -0.217 & 0.062 & 0.002 & -0.126 & 0.166 & 0.058 \\
\hline DB2Vec Similarity & 0.743 & 0.734 & 0.635 & 0.669 & 0.628 & 0.692 \\
\hline DB2Vec Relatedness & 0.811 & 0.778 & 0.711 & 0.658 & 0.670 & 0.736 \\
\hline
\end{tabular}

\subsection{Document similarity}

To evaluate the document similarity approach, we use the LP50 dataset [37], namely a collection of 50 news articles from the Australian Broadcasting Corporation $(\mathrm{ABC})$, which were pairwise annotated with similarity rating on a Likert scale from 1 (very different) to 5 (very similar) by 8 to 12 different human annotators. To obtain the final similarity judgments, the scores of all annotators are averaged. As a evaluation metrics we use Pearson's linear correlation coefficient and Spearman's rank correlation plus their harmonic mean.

Again, we first evaluate each of the RDF2Vec models separately. Table 9 shows document similarity results. As for the entity relatedness, the results show that the skip-gram models built on DBpedia with 8 hops lead to the best performances.

Furthermore, we compare our approach to several state-of-the-art graph-based document similarity approaches:

- TF-IDF: Distributional baseline algorithm.

- AnnOv: Similarity score based on annotation overlap that corresponds to traversal entity similarity with radius 0 , as described in [62].

- Explicit Semantic Analysis (ESA) [21].

- GED: semantic similarity using a Graph Edit Distance based measure [82].

- Salient Semantic Analysis (SSA), Latent Semantic Analysis (LSA) [27].

- Graph-based Semantic Similarity (GBSS) [62]. 
Table 9

Document similarity results - Pearson's linear correlation coefficient ( $r$ ) Spearman's rank correlation $(\rho)$ and their harmonic mean $\mu$

\begin{tabular}{lccc}
\hline Model & $r$ & $\rho$ & $\mu$ \\
\hline DB2vec SG 200w 200v 4d & 0.608 & 0.448 & 0.516 \\
DB2vec CBOW 200w 200v 4d & 0.562 & 0.480 & 0.518 \\
DB2vec CBOW 500w 200v 4d & 0.681 & 0.535 & 0.599 \\
DB2vec CBOW 500w 500v 4d & 0.677 & 0.530 & 0.594 \\
DB2vec SG 500w 200v 4d & 0.639 & 0.520 & 0.573 \\
DB2vec SG 500w 500v 4d & 0.641 & 0.516 & 0.572 \\
DB2vec CBOW 500w 200v 8d & 0.658 & 0.491 & 0.562 \\
DB2vec CBOW 500w 500v 8d & 0.683 & 0.512 & 0.586 \\
DB2vec SG 500w 200v 8d & $\mathbf{0 . 7 0 8}$ & $\mathbf{0 . 5 5 6}$ & $\mathbf{0 . 6 2 3}$ \\
DB2vec SG 500w 500v 8d & 0.686 & 0.527 & 0.596 \\
WD2vec CBOW 200w 200v 4d & 0.568 & 0.383 & 0.458 \\
WD2vec CBOW 200w 500v 4d & 0.593 & 0.386 & 0.467 \\
WD2vec SG 200w 200v 4d & 0.606 & 0.385 & 0.471 \\
WD2vec SG 200w 500v 4d & 0.613 & 0.343 & 0.440 \\
Wiki2vec & 0.662 & 0.513 & 0.578 \\
DB_TransE & 0.565 & 0.432 & 0.490 \\
DB_TransH & 0.570 & 0.452 & 0.504 \\
DB_TransR & 0.578 & 0.461 & 0.513 \\
\hline
\end{tabular}

Table 10

Comparison of the document similarity approach to the related work

\begin{tabular}{lccc}
\hline Approach & $r$ & $\rho$ & $\mu$ \\
\hline TF-IDF & 0.398 & 0.224 & 0.287 \\
AnnOv & 0.590 & 0.460 & 0.517 \\
LSA & 0.696 & 0.463 & 0.556 \\
SSA & 0.684 & 0.488 & 0.570 \\
GED & 0.630 & 1 & 1 \\
ESA & 0.656 & 0.510 & 0.574 \\
GBSS & 0.704 & 0.519 & 0.598 \\
DB2Vec & $\mathbf{0 . 7 0 8}$ & $\mathbf{0 . 5 5 6}$ & $\mathbf{0 . 6 2 3}$ \\
\hline
\end{tabular}

The results for the related approaches were copied from the respective papers, except for ESA, which was copied from [62], where it is calculated via public ESA REST endpoint. ${ }^{17}$ The results, shown in Table 10, show that our document similarity approach outperforms all of the related approaches for both Pearson's linear correlation coefficient and Spearman's rank correlation, as well as their harmonic mean.

We do not compare our approach to the machinelearning approach proposed by Huang et al. [30], because that approach is a supervised one, which is tai-

\footnotetext{
${ }^{17}$ http://vmdeb20.deri.ie:8890/esaservice
}

lored towards the dataset, whereas ours (as well as the others we compare to) are unsupervised.

\section{Recommender systems}

As discussed in Section 2.3, the Linked Open Data (LOD) initiative [5] has opened new interesting possibilities to realize better recommendation approaches. Given that the items to be recommended are linked to a LOD dataset, information from LOD can be exploited to determine which items are considered to be similar to the ones that the user has consumed in the past, allowing to discover hidden information and implicit relations between objects. While LOD is rich in high quality data, it is still challenging to find an effective and efficient way of exploiting the knowledge for content-based recommendations. So far, most of the proposed approaches in the literature are supervised or semi-supervised, which means they cannot work without human interaction. New challenges, and at the same time new opportunities, arise from unsupervised approaches for feature learning.

RDF graph embeddings are a promising way to approach those challenges and build content-based recommender systems. As for entity similarity in the previous section, the cosine similarity between the latent vectors representing the items can be interpreted as a measure of reciprocal proximity and then exploited to produce recommendations.

In this section, we explore the development of a hybrid RS, leveraging the latent features extracted with RDF2Vec. The hybrid system takes advantage of both RDF graph embeddings and Factorization Machines (FMs) [68], an effective method combining Support Vector Machines with factorization models.

\subsection{Factorization machines}

Factorization Machines (FMs) [68] are a general predictor model which rely on the advantages of Support Vector Machines (SVMs) and factorization models. SVMs have been successfully applied in many classification and regression problems but they have been proved to be not effective in those settings like collaborative filtering which are characterized by huge sparsity. This happens since in sparse settings there is not enough data to estimate a "dense" factorization as SVMs would do. FM use a factorized parametrization, breaking the independence of the interaction parameters by factorizing them. The difference clearly 
emerges when the model equations of degree 2 for a factorization machine and for a SVM are directly compared. Being aware that in a prediction task the general goal is to estimate a function $y: \mathcal{R}^{n} \rightarrow T$, from a real valued feature vector $x \in \mathcal{R}^{n}$ to a target domain $T$, the model equation [68] for a FM of degree 2 is given by:

$$
\begin{aligned}
\hat{y}(x)= & w_{0}+\sum_{i=1}^{n} w_{i} x_{i} \\
& +\sum_{i=1}^{n} \sum_{j=i+1}^{n}\left(v_{i}^{T} v_{j}\right) x_{i} x_{j}
\end{aligned}
$$

where $\hat{y}$ represents the estimate of function $y$. In (10) the parameters to be estimated are the global bias $w_{0} \in \mathcal{R}$, the vector $w=\left[w_{1}, \ldots, w_{n}\right] \in \mathcal{R}^{n}$ whose component $w_{i}$ models the strength of the $i_{\text {th }}$ variable and a matrix $V \in \mathcal{R}^{n \times k}$, which describes the $i_{\text {th }}$ variable with its $i_{\text {th }}$ row $v_{i}$, i.e., with a vector of size $k$, where $k$ is a hyperparameter defining the factorization dimensionality. On the other hand the model equation for a SVM of the same degree 2 is defined as:

$$
\begin{aligned}
\hat{y}(x)= & w_{0}+\sqrt{2} \sum_{i=1}^{n} w_{i} x_{i}+\sum_{i=1}^{n} w_{i, i}^{(2)} x_{i}^{2} \\
& +\sqrt{2} \sum_{i=1}^{n} \sum_{j=i+1}^{n} w_{i, j}^{(2)} x_{i} x_{j}
\end{aligned}
$$

with model parameters $w_{0} \in \mathcal{R}, w \in \mathcal{R}^{n}$ and the symmetric matrix $W^{(2)} \in \mathcal{R}^{n \times n}$. From the comparison of equations (10) and (11) we can realize that both model all nested interactions up to degree 2 but with a fundamental difference: the estimation of the interactions between variables in the SVM model is done directly and independently with $w_{i, j}$ whereas it is parametrized in the FM model. In other words, parameters $w_{i, j}$ and $w_{i, l}$ will be completely independent in the SVM case while parameters $v_{i}^{T} v_{j}$ and $v_{i}^{T} v_{l}$ overlap thanks to the shared parameter $v_{i}$.

The strength of FMs models is also due to linear complexity [68] and due to the fact that they can be optimized in the primal and they do not rely on support vectors like SVMs. Finally, [68] shows that many of the most successful approaches for the task of collaborative filtering are de facto subsumed by FMs, e.g., Matrix Factorization [80,86] and SVD++ [34].

\subsection{Experiments}

We evaluate different variants of our approach on three datasets, and compare them to common ap- proaches for creating content-based item representations from LOD, as well as to state of the art collaborative and hybrid approaches. Furthermore, we investigate the use of two different LOD datasets as background knowledge, i.e., DBpedia and Wikidata.

\subsubsection{Datasets}

In order to test the effectiveness of vector space embeddings for the recommendation task, we have performed an extensive evaluation in terms of ranking accuracy on three datasets belonging to different domains, i.e., Movielens ${ }^{18}$ for movies, LibraryThing ${ }^{19}$ for books, and Last. $\mathrm{fm}^{20}$ for music. The first dataset, Movielens $1 \mathrm{M}$, contains 1 million 15 stars ratings from 6,040 users on 3,952 movies. The dataset LibraryThing contains more than 2 millions ratings from 7,279 users on 37,232 books. As there are many duplicated ratings in the dataset, which occur when a user has rated the same item more than once, her last rating is selected. The unique ratings are 749,401 , in the range from 1 to 10 . Both Movielens and LibraryThing datasets contain explicit ratings, and to test the approach also on implicit feedbacks, a third dataset built on the top of the Last. fm music system is considered. Last. fm contains 92,834 interactions between 1,892 users and 17,632 musical artists. Each interaction is annotated with the corresponding listening count.

The original datasets are enriched with background information using the item mapping and linking to DBpedia technique described in [60], whose dump is available publicly. ${ }^{21}$ Since not all the items have a corresponding resource in DBpedia, after the mapping, the versions of Movielens, LibraryThing and Last.fm datasets contain 3,883 movies, 11,695 books, and 11,180 musical artists, respectively.

The datasets are finally preprocessed to guarantee a fair comparison with the state of the art approaches described in [57]. Here, the authors propose to (i) remove popularity biases from the evaluation not considering the top $1 \%$ most popular items, (ii) reduce the sparsity of Movielens dataset in order to have at least a sparser test dataset and (iii) remove from LibraryThing and Last. fm users with less than five ratings and items rated less than five times. The final statistics on the three datasets are reported in Table 11.

\footnotetext{
${ }^{18} \mathrm{http}: / /$ grouplens.org/datasets/movielens/

${ }^{19} \mathrm{https} / / / \mathrm{www}$. librarything.com/

${ }^{20} \mathrm{http}: / / \mathrm{www} \cdot$ lastfm.com

${ }^{21}$ https://github.com/sisinflab/LODrecsys-datasets
} 
Table 11

Statistics about the three datasets

\begin{tabular}{lccc}
\hline & Movielens & LibraryThing & Last.fm \\
\hline Number of users & 4,186 & 7,149 & 1,875 \\
Number of items & 3,196 & 4,541 & 2,432 \\
Number of ratings & 822,597 & 352,123 & 44,981 \\
Data sparsity & $93.85 \%$ & $98.90 \%$ & $99.01 \%$ \\
\hline
\end{tabular}

\subsubsection{Evaluation protocol}

The ranking setting for the recommendation task consists of producing a ranked list of items to suggest to the user and in practical situations turns into the socalled top- $N$ recommendation task, where just a cutoff of the ranked list of size $N$ is provided to the user. This setting has recently replaced the rating prediction, because of the increasing awareness that the user is not interested in an accurate prediction of the item rating, but is looking for a (limited) list of items extracted from the pool of available ones.

As evaluation ranking protocol for our comparison, we adopted the all unrated items methodology presented in [87] and already used in [57]. This methodology asks to predict a score for each item not rated by a user, irrespective of the existence of an actual rating, and to compare the recommendation list with the test set.

The metrics involved in the experimental comparison are three well-known ranking measures for recommendation accuracy, i.e., precision, recall, F-score (F1) and $\mathrm{nDCG}$.

- precision@N [70] represents the fraction of relevant items in the top- $N$ recommendations.

- recall@N [70] indicates the fraction of relevant items, in the user test set, occurring in the top- $N$ list. As relevance threshold, we set 4 for Movielens and 8 for LibraryThing, as previously done in [57].

- The $F 1$ score [70], i.e., the harmonic mean of precision and recall, is also pointed out to be thorough. Although precision and recall are good indicators to evaluate the accuracy of a recommendation engine, they are not rank-sensitive.

- The normalized Discounted Cumulative Gain $n D C G @ N$ [3] instead takes into account also the position in the recommendation list, being defined as

$$
\begin{aligned}
& \text { nDCG@N } N \\
& =\frac{1}{\mathrm{iDCG}} \cdot \sum_{i=1}^{N} \frac{2^{\operatorname{rel}(u, i)}-1}{\log _{2}(1+i)}
\end{aligned}
$$

where $\operatorname{rel}(u, i)$ is a Boolean function representing the relevance of item $i$ for user $u$ and iDCG is a normalization factor that sets nDCG@ $N$ value to 1 when an ideal ranking is returned [3].

As suggested in [87] and set up in [57], in the computation of nDCG@ $N$ we fixed a default "neutral" value for those items with no ratings, i.e., 3 for Movielens and 5 for LibraryThing.

All the results have been computed @10,i.e., considering the top-10 list recommended to each user and then averaging across all users.

\subsubsection{Experimental setup}

The target of this experimental section is two-fold. On the one hand, we show that the proposed graph embeddings technique outperforms other strategies for feature creation. On the other hand, we combine it with a hybrid RS and compare the resulting system's relative performances with state of the art approaches. The first goal is pursued by implementing an itembased K-Nearest Neighbor method, hereafter denoted as ItemKNN, with cosine similarity among features vectors. As data mining features, the approaches based on direct relations and graph substructures, as detailed in Section 5.1, are considered.

For what concerns the comparison against some of the most promising collaborative and hybrid approaches currently available, we consider the results recently published in [57]. There, the authors show that the best effectivenesses in terms of ranking accuracy is reached by the following algorithms:

- SLIM [54] is a Sparse LInear Method for top- $N$ recommendation that learns a sparse coefficient matrix for the items involved in the system by only relying on the users purchase/ratings profile and by solving a L1-norm and L2-norm regularized optimization problem.

- BPR-SSLIM is a Sparse LInear Method using item Side information (SSLIM) [55] and the Bayesian Personalized Ranking (BPR) optimization criterion [69].

- SPRank [57] is a novel hybrid recommender system that solves the top- $N$ recommendation problem in a learning to rank fashion, exploiting the freely available knowledge in the Linked Open Data to build semantic path-based features.

In [57], it has been proved that SPRank is able to outperform SLIM and BPR-SSLIM (at least according to Precision and $\mathrm{nDCG}$ ) in those context characterized by higher sparsity, where reasonably the con- 
tribution of the content is more essential. For the sake of completeness, it is necessary to remind that in [57] the aforementioned approaches are tested against well established collaborative algorithms for rating prediction, e.g., Biased Matrix Factorization [35], and for items ranking, such as Bayesian Personalized Ranking [69] and Soft Margin Ranking Matrix Factorization [93]. Furthermore, two algorithms that work on heterogeneous information networks are also involved in the comparison, i.e., PathRank [38], an extension of Personalized PageRank for heterogeneous networks, and HeteRec [96], which represents the connectivity between users and items through a matrix factorization algorithm that uses meta-path based latent features.

\subsection{Results}

We present the results by a purely content-based RS and a hybrid RS using RDF2Vec.

\subsubsection{Content-based approach}

The first experiment aims to point out the validity of the proposed graph embeddings technique for feature generation in the context of content-based RS, and relies on a relatively simple recommendation algorithm, i.e., the item-based K-Nearest Neighbor approach [70] with cosine similarity. Formally, this method evaluates the closeness of items through cosine similarity between the corresponding features vectors and then selects a subset of those - the neighbors - for each item, that will be used to estimate the rating of user $u$ for a new item $i$ as follows:

$$
\begin{aligned}
r^{*}(u, i) & \\
= & \frac{\sum_{j \in \text { ratedItems }(u)} \operatorname{cosineSim}(j, i) \cdot r_{u, j}}{\sum_{j \in \text { rateditems }(u)}|\operatorname{cosineSim}(j, i)|}
\end{aligned}
$$

where ratedItems $(u)$ is the set of items already evaluated by user $u, r_{u, j}$ indicates the rating for item $j$ by user $u$ and cosineSim $(j, i)$ is the cosine similarity score between items $j$ and $i$. In our experiments, the size of the considered neighbourhood is limited to 5 .

The vector representation of items made of latent features, output of the RDF graph embeddings with graph walks, is evaluated against all the other data mining features listed in Section 5.1. Tables 12, 13 and 14 contain the values of precision, recall, F-score (F1) and nDCG, respectively for Movielens, LibraryThing and Last.fm. The computation of recom- mendations has been done with the publicly available library RankSys. ${ }^{22}$

The first conclusion that can be drawn from $\mathrm{Ta}-$ bles 12,13 and 14 is that the best approach for all datasets is retrieved with a skip-gram model, 500 walks per entity and with a size of 200 for vectors built upon DBpedia. Altough on Movielens, the highest value of precision is achived using vector size of 500, the size 200 is prevalent according to the F1 measure. A substantial difference concerns the exploratory depth of the random walks, since for Movielens the results related to a depth of 4 outperform those computed with a depth of 8 , while the tendency is reversed for both LibraryThing and Last. fm. Secondly, the advantage of the Skip-Gram model over CBOW is a constant both on DBpedia and Wikidata and is particularly evident when the model involves longer random walks, i.e., with depth 8 .

Comparing the LOD datasets, it clearly emerges that DBpedia lets to gain higher values than Wikidata for each metric involved, but it turns out that Wikidata is quite effective on LibraryThing, where the skipgram vectors with depth of 4 exceed the corresponding DBpedia vectors. Again, for the domains at hand books, movies, and musical works and artists - DBpedia has a better level of detail (the average in Table 5 is caused by the larger number of books in Wikidata, where the long tail may be described at a lower level of detail [71], but the head entities in the LibraryThing dataset are described very well).

Moving to the features extracted from direct relations, the contribution of the "categories" stands clearly out, together with relations-values "rel-vals", especially when just incoming relations are considered: these features allow to achieve better results than the approaches based on translating embeddings, i.e., DB_TransE, DB_TransH and DB_TransR. The use of methods based on kernels for features extraction, i.e., WC_4 and WL_2_2 approaches, seems not to provide significant advantages to the recommendation algorithm.

To point out that the latent features built upon RDF graph are able to capture its structure, placing closely semantically similar items, some examples of the neighbouring sets retrieved using the graph embeddings technique are provided. These sets are directly exploited by the ItemKNN algorithm to produce recommendations. Table 15 is related to movies and to

\footnotetext{
${ }^{22} \mathrm{http}: / /$ ranksys.org/
} 
Results of the ItemKNN approach on Movielens dataset with reference to different computation of features

\begin{tabular}{|c|c|c|c|c|}
\hline Strategy & Precision & Recall & F1 & $\mathrm{nDCG}$ \\
\hline types & 0.00313 & 0.00145 & 0.00198 & 0.28864 \\
\hline categories & 0.0305 & 0.02093 & 0.02482 & 0.30444 \\
\hline rel in & 0.01122 & 0.00589 & 0.0077 & 0.29183 \\
\hline rel out & 0.02844 & 0.01607 & 0.02053 & 0.30274 \\
\hline rel in \& out & 0.02852 & 0.01566 & 0.02021 & 0.3006 \\
\hline rel-vals in & 0.03883 & 0.02293 & 0.02882 & 0.29411 \\
\hline rel-vals out & 0.01279 & 0.00971 & 0.011 & 0.29378 \\
\hline rel-vals in \& out & 0.01174 & 0.00913 & 0.01027 & 0.29333 \\
\hline WC_4 & 0.00684 & 0.00343 & 0.0045 & 0.29032 \\
\hline WL_2_2 & 0.00601 & 0.00288 & 0.00389 & 0.28977 \\
\hline DB_TransE & 0.03047 & 0.01411 & 0.01928 & 0.30385 \\
\hline DB_TransH & 0.02649 & 0.01187 & 0.01639 & 0.30016 \\
\hline DB_TransR & 0.00941 & 0.0043 & 0.0059 & 0.29216 \\
\hline DB2vec SG 200w 200v 4d & 0.05423 & 0.02693 & 0.03598 & 0.31676 \\
\hline DB2vec CBOW 200w 200v 4d & 0.03475 & 0.01637 & 0.02225 & 0.30426 \\
\hline DB2vec CBOW 500w 200v 4d & 0.03893 & 0.02167 & 0.02784 & 0.30782 \\
\hline DB2vec CBOW 500w 500v 4d & 0.03663 & 0.02088 & 0.02659 & 0.30557 \\
\hline DB2vec SG 500w 200v 4d & 0.05681 & 0.03119 & 0.04027 & 0.31828 \\
\hline DB2vec SG 500w 500v 4d & 0.05786 & 0.0304 & 0.03985 & 0.31726 \\
\hline DB2vec CBOW 500w 200v 8d & 0.01064 & 0.00548 & 0.00723 & 0.29245 \\
\hline DB2vec CBOW 500w 500v 8d & 0.01137 & 0.00567 & 0.00756 & 0.29289 \\
\hline DB2vec SG 500w 200v 8d & 0.04424 & 0.02693 & 0.03347 & 0.30997 \\
\hline DB2vec SG 500w 500v 8d & 0.02191 & 0.01478 & 0.01765 & 0.29863 \\
\hline WD2vec CBOW 200w 200v 4d & 0.01217 & 0.00596 & 0.00800 & 0.29362 \\
\hline WD2vec CBOW 200w 500v 4d & 0.01027 & 0.00427 & 0.0060 & 0.29211 \\
\hline WD2vec SG 200w 200v 4d & 0.02902 & 0.01479 & 0.01959 & 0.30189 \\
\hline WD2vec SG 200w 500v 4d & 0.02644 & 0.01246 & 0.01693 & 0.29967 \\
\hline
\end{tabular}

the strategy "DB2vec SG 500w 200v 4d", and displays that neighboring items are highly relevant and close to the query item, i.e., the item for which neighbors are searched for. Figure 4 depicts the 2D PCA projection of the movies in that table, showing that similar movies are actually projected closely to each other.

\subsubsection{Hybrid approach}

To second goal of our experiments is to show that a hybrid recommender system, leveraging the item content built with the graph embeddings technique, can compete against some of the most promising state of the art approaches for recommendation. A hybrid RS based on Factorization Machines is utilized, namely the ranking factorization machine algorithm available in the Graphlab recommender toolkit. ${ }^{23}$ This algo-

\footnotetext{
${ }^{23}$ https://dato.com/products/create/docs/graphlab.toolkits. recommender.html
}

rithm allows to define both users and items side data. Grounding on the capability to automatically cluster semantically similar items pointed out by the experiments of the previous section, the additional information added to each item, as side data, is the list of its 5 nearest neighbors. The tuning of the hyperparameters (specifically the numbers of latent factors and the maximum number of iterations) has been carried out through cross-validation on a validation set obtained selecting the $15 \%$ of the ratings of each user from the training set. The same choice for cross validation has been adopted for the competing recommendations algorithms, as already done in [57].

Tables 16, 17, and 18 report, respectively for Movie-lens, LibraryThing and Last.fm, the most promising approach according to the analysis conducted with the ItemKNN algorithm in Section 7.3.1 (Tables from 12 to 14) and the competing 
Table 13

\begin{tabular}{|c|c|c|c|c|}
\hline Strategy & Precision & Recall & $\mathrm{F} 1$ & $\mathrm{nDCG}$ \\
\hline types & 0.01854 & 0.04535 & 0.02631 & 0.16064 \\
\hline categories & 0.06662 & 0.15258 & 0.09274 & 0.23733 \\
\hline rel in & 0.04577 & 0.10219 & 0.06322 & 0.20196 \\
\hline rel out & 0.04118 & 0.09055 & 0.05661 & 0.19449 \\
\hline rel in \& out & 0.04531 & 0.10165 & 0.06268 & 0.20115 \\
\hline rel-vals in & 0.06176 & 0.14101 & 0.08589 & 0.22574 \\
\hline rel-vals out & 0.06163 & 0.13763 & 0.08513 & 0.22826 \\
\hline rel-vals in \& out & 0.06087 & 0.13662 & 0.08421 & 0.22615 \\
\hline WC_4 & 0.00159 & 0.00306 & 0.00209 & 0.12858 \\
\hline WL_2_2 & 0.00155 & 0.00389 & 0.00221 & 0.12937 \\
\hline DB_TransE & 0.01819 & 0.04705 & 0.02623 & 0.1585 \\
\hline DB_TransH & 0.01466 & 0.03997 & 0.02145 & 0.15331 \\
\hline DB_TransR & 0.00162 & 0.00341 & 0.00219 & 0.12947 \\
\hline DB2vec SG 200w 200v 4d & 0.00442 & 0.00942 & 0.00601 & 0.13502 \\
\hline DB2vec CBOW 200w 200v 4d & 0.00466 & 0.00933 & 0.00621 & 0.13595 \\
\hline DB2vec CBOW 500w 200v 4d & 0.05127 & 0.11777 & 0.07143 & 0.21244 \\
\hline DB2vec CBOW 500w 500v 4d & 0.05065 & 0.11557 & 0.07043 & 0.21039 \\
\hline DB2vec SG 500w 200v 4d & 0.05719 & 0.12763 & 0.07898 & 0.2205 \\
\hline DB2vec SG 500w 500v 4d & 0.05811 & 0.12864 & 0.08005 & 0.22116 \\
\hline DB2vec CBOW 500w 200v 8d & 0.00836 & 0.02334 & 0.01231 & 0.14147 \\
\hline DB2vec CBOW 500w 500v 8d & 0.00813 & 0.02335 & 0.01206 & 0.14257 \\
\hline DB2vec SG 500w 200v 8d & 0.07681 & 0.17769 & 0.10725 & 0.25234 \\
\hline DB2vec SG 500w 500v 8d & 0.07446 & 0.1743 & 0.10434 & 0.24809 \\
\hline WD2vec CBOW 200w 200v 4d & 0.00537 & 0.01084 & 0.00718 & 0.13524 \\
\hline WD2vec CBOW 200w 500v 4d & 0.00444 & 0.00984 & 0.00611 & 0.13428 \\
\hline WD2vec SG 200w 200v 4d & 0.06416 & 0.14565 & 0.08907 & 0.23309 \\
\hline WD2vec SG 200w 500v 4d & 0.06031 & 0.14194 & 0.08465 & 0.22752 \\
\hline
\end{tabular}

approaches SPRank, SLIM and BPR-SSLIM. ${ }^{24}$ For BPR-SSLIM, the resources directly connected to the items are used as item side data, as in [57]. The prefix " $\mathcal{H}$-" is used as a notation for the hybrid RS grounding on the relative feature vectors. SLIM and BPR-SSLIM are implemented through the publicly available software library MyMediaLite ${ }^{25}$ [22], while for SPRank, the implementation provided by its authors, available publicly, ${ }^{26}$ is used. We repeated the experiments three times for each datasets and then averaged the results across the three runs (reporting also the standard deviation).

\footnotetext{
${ }^{24}$ The complete version of these tables and the code for implementing the hybrid system are available at http://sisinflab.poliba.it/ recommender-systems/hybridSystemRDF2Vec.html.

${ }^{25}$ http://www.mymedialite.net

${ }^{26}$ https://github.com/sisinflab/lodreclib
}

Looking at Tables 16, 17 and 18, we can observe performance improvements on all datasets and according to each of the metrics involved, with the only exception of the precision on the Last. fm dataset. In particular, on Movielens, the hybrid SPRank cannot compete with the collaborative method SLIM and to its extended version with side data, BPR-SSLIM, probably because of the key contribution of collaborative information on this dataset. In fact, Movielens is the denser dataset in the comparison, containing only experienced users with more than fifty ratings. Nevertheless, the approach " $\mathcal{H}$-DB2vec SG 500w $200 \mathrm{v} 4 \mathrm{~d}$ " outperforms the competing ones, reasonably with the key contribution of factorization models in FM. On the other datasets, whose sparsity better reproduces real-world scenarios, the contribution of content appears more determinant. This emerges in par-

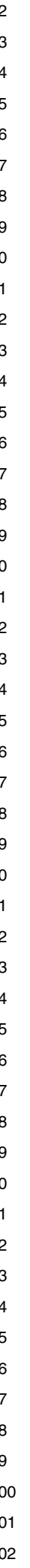


Table 14

Results of the ItemKNN approach on Last. fm with reference to different computation of features

\begin{tabular}{|c|c|c|c|c|}
\hline Strategy & Precision & Recall & $\mathrm{F} 1$ & $\mathrm{nDCG}$ \\
\hline types & 0.00525 & 0.03256 & 0.009 & 0.01826 \\
\hline categories & 0.01762 & 0.09889 & 0.02991 & 0.06023 \\
\hline rel in & 0.00625 & 0.03625 & 0.01066 & 0.02042 \\
\hline rel out & 0.00519 & 0.02757 & 0.00873 & 0.01733 \\
\hline rel in \& out & 0.00718 & 0.04205 & 0.01226 & 0.02567 \\
\hline rel-vals in & 0.0185 & 0.10502 & 0.03145 & 0.06733 \\
\hline rel-vals out & 0.00805 & 0.04585 & 0.01369 & 0.0248 \\
\hline rel-vals in \& out & 0.00339 & 0.01774 & 0.00569 & 0.00982 \\
\hline WC_4 & 0.00086 & 0.00401 & 0.00141 & 0.00241 \\
\hline WL_2_2 & 0.00086 & 0.00344 & 0.00137 & 0.00215 \\
\hline DB_TransE & 0.01117 & 0.06078 & 0.01887 & 0.03953 \\
\hline DB_TransH & 0.01409 & 0.07928 & 0.02392 & 0.04881 \\
\hline DB_TransR & 0.0011 & 0.00523 & 0.00181 & 0.00381 \\
\hline DB2vec SG 200w 200v 4d & 0.01 & 0.05498 & 0.01692 & 0.02911 \\
\hline DB2vec CBOW 200w 200v 4d & 0.00929 & 0.05227 & 0.01577 & 0.03288 \\
\hline DB2vec CBOW 500w 200v 4d & 0.01749 & 0.09915 & 0.02973 & 0.06435 \\
\hline DB2vec CBOW 500w 500v 4d & 0.01769 & 0.10016 & 0.03006 & 0.06404 \\
\hline DB2vec SG 500w 200v 4d & 0.02015 & 0.11109 & 0.03411 & 0.07232 \\
\hline DB2vec SG 500w 500v 4d & 0.02001 & 0.10978 & 0.03385 & 0.07448 \\
\hline DB2vec CBOW 500w 200v 8d & 0.00944 & 0.05349 & 0.01604 & 0.03311 \\
\hline DB2vec CBOW 500w 500v 8d & 0.00964 & 0.0563 & 0.01646 & 0.03166 \\
\hline DB2vec SG 500w 200v 8d & 0.0234 & 0.1359 & 0.03992 & 0.08719 \\
\hline DB2vec SG 500w 500v 8d & 0.02088 & 0.12248 & 0.03567 & 0.07789 \\
\hline WD2vec CBOW 200w 200v 4d & 0.00133 & 0.00785 & 0.00227 & 0.00382 \\
\hline WD2vec CBOW 200w 500v 4d & 0.001 & 0.00532 & 0.00168 & 0.00408 \\
\hline WD2vec SG 200w 200v 4d & 0.00612 & 0.03388 & 0.01036 & 0.02157 \\
\hline WD2vec SG 200w 500v 4d & 0.00658 & 0.03932 & 0.01127 & 0.02382 \\
\hline
\end{tabular}

Table 15

Examples of K-Nearest Neighbor sets on Movielens

\begin{tabular}{ll}
\hline Query Movie & \multicolumn{1}{c}{ K Nearest Neighbours } \\
\hline Batman & Batman Forever, Batman \\
& Returns, Batman \& Robin, \\
& Superman IV: The Quest \\
& for Peace, Dick Tracy \\
& Cinderella, Dumbo, 101 \\
& Dalmatians, Pinocchio, \\
Lambi & Lady and the Tramp \\
& Star Trek VI: The \\
& Undiscovered Country, Star \\
Star Trek: Generations & Trek: Insurrection, Star \\
& Trek III: The Search for \\
& Spock, Star Trek V: The \\
& Final Frontier, Star Trek: \\
& First Contact \\
\hline
\end{tabular}

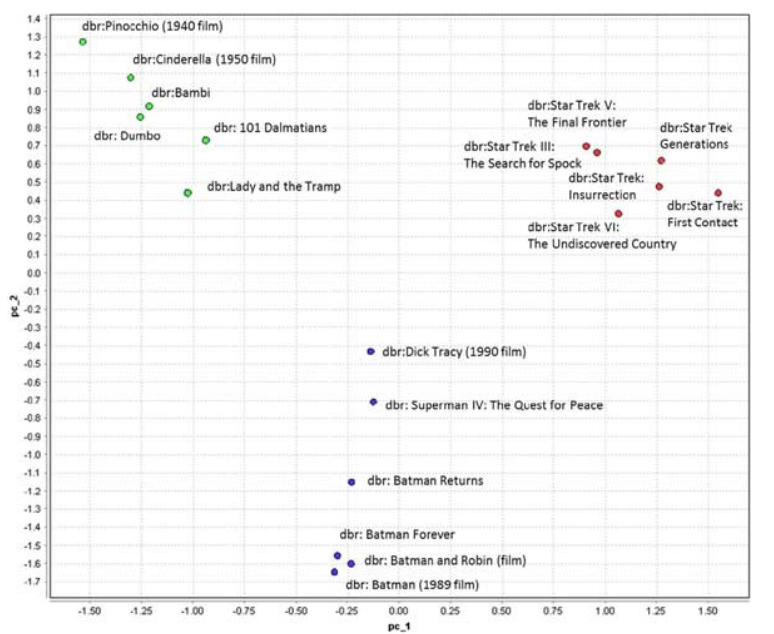

Fig. 4. Two-dimensional PCA projection of the 200-dimensional Skip-Gram vectors of movies in Table 15. 
Table 16

\begin{tabular}{|c|c|c|c|c|}
\hline Strategy & Precision & Recall & F1 & $\mathrm{nDCG}$ \\
\hline $\mathcal{H}$-DB2vec SG 500w 200v 4d & $\mathbf{0 . 2 6 9 8} \pm 0.0012$ & $\mathbf{0 . 1 4 8 0} \pm 0.0013$ & $\mathbf{0 . 1 9 1 1} \pm 0.0013$ & $\mathbf{0 . 4 7 8 2} \pm 0.0007$ \\
\hline SPRank & $0.1613^{*}$ & $0.0785^{*}$ & 0.1056 & $0.4056^{*}$ \\
\hline SLIM & $0.2631^{*}$ & 0.1469 & 0.1885 & $0.4597^{*}$ \\
\hline BPR-SSLIM & $0.2636^{*}$ & $0.1439^{* *}$ & 0.1861 & $0.4674^{*}$ \\
\hline
\end{tabular}

Table 17

Comparative results on LibraryThing dataset. The differences between " $\mathcal{H}$-DB2vec SG 500w 200v 8d" and the other methods are statistically significant according to the Wilcoxon signed rank with $p<0.001$. The scores for " $\mathcal{H}$-DB2vec SG 500w 200v 8d", averaged over three runs, are reported with the standard deviation

\begin{tabular}{|c|c|c|c|c|}
\hline Strategy & Precision & Recall & $\mathrm{F} 1$ & $\mathrm{nDCG}$ \\
\hline 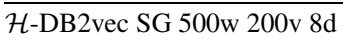 & $\mathbf{0 . 1 0 6 1} \pm 0.0016$ & $\mathbf{0 . 2 4 1} \pm 0.0036$ & $\mathbf{0 . 1 4 7 3} \pm 0.0022$ & $\mathbf{0 . 3 1 2 7} \pm 0.005$ \\
\hline SPRank & 0.1019 & 0.2232 & 0.1399 & 0.3068 \\
\hline SLIM & 0.0543 & 0.0988 & 0.07 & 0.2317 \\
\hline BPR-SSLIM & 0.0962 & 0.2328 & 0.1361 & 0.3051 \\
\hline
\end{tabular}

Table 18

Comparative results on Last. fm dataset. The differences between " $\mathcal{H}$-DB2vec SG 500w 200v 8d" and the other methods are statistically significant according to the Wilcoxon signed rank with $p<0.001$. The scores for " $\mathcal{H}$-DB2vec SG 500w 200v 8d", averaged over three runs, are reported with the standard deviation

\begin{tabular}{|c|c|c|c|c|}
\hline Strategy & Precision & Recall & $\mathrm{F} 1$ & nDCG \\
\hline 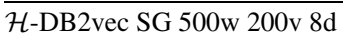 & $0.0607 \pm 0.0021$ & $\mathbf{0 . 3 4 0 8} \pm 0.0143$ & $0.103 \pm 0.003$ & $0.2266 \pm 0.01$ \\
\hline SPRank & 0.0841 & 0.2043 & 0.1191 & 0.1918 \\
\hline SLIM & 0.0723 & 0.1548 & 0.0985 & 0.1395 \\
\hline BPR-SSLIM & 0.0465 & 0.2649 & 0.0791 & 0.1759 \\
\hline
\end{tabular}

ticular on LibraryThing, where BPR-SSLIM and SPRank overtake SLIM and " $\mathcal{H}$-DB2vec SG 500w 200v $8 \mathrm{~d}$ " improvesthe results even further. On the third dataset, i.e., Last. fm, the hybrid approaches generally perform better than SLIM (except on precision for BPR-SSLIM) and the approach based on graph embeddings reports a further enhancement in recall and nDCG. The results are statistically significant according to the Wilcoxon signed rank with $p<0.001$, for less than a different specification. In conclusion, the choice of using Factorization Machines and of building item side data by means of the RDF graph embeddings technique, turns out to be favorable for fostering accuracy in general and getting improvements, on every dataset, on the rank-sensitive metric nDCG. We remind that the importance of providing to the user an opportunely ranked list of recommendations is nowadays recognized as fundamental in the RS research community.

\section{Conclusion and outlook}

In this paper, we have presented RDF2Vec, an approach for learning latent numerical representations of entities in RDF graphs. In this approach, we first convert the RDF graphs in a set of sequences using two strategies, Weisfeiler-Lehman Subtree RDF Graph Kernels and graph walks, which are then used to build neural language models. The evaluation shows that such entity representations could be used in three different tasks. In each of those tasks, they were capable of outperforming standard feature generation approaches, i.e., approaches that turn (subsets of) RDF graphs into propositional models.

We have explored different variants of building embedding models. While there is no universally best performing approach, we can observe some trends. With construction of sequences, kernel transformations lead to better results than (random) walks. However, they respect to the first step of the transformation, i.e., the 
do not scale well to large-scale knowledge graphs, such as DBpedia or Wikidata. With respect to the second step, i.e., the actual computation of the embeddings, we have observed that Skip-Gram (SG) models in most cases outperform Continuous-Bag-of-Words (CBOW) models. The other characteristics of the models (e.g., the dimensionality of the embedding space) show less clear trends towards an optimal setting.

While constructing a vector space embedding for a large-scale knowledge graph, such as DBpedia or Wikidata, can be computationally expensive, we have shown that this step has to be taken only once, as the embeddings can be reused on various tasks. This is particularly interesting for such cross-domain knowledge graphs, which can be used in a variety of scenarios and applications.

For the moment, we have defined some constraints for the construction of the embeddings. We do not use literal values, and we do not particularly distinguish between the schema and the data level of a graph. The former constraint has some limitations, e.g., when it comes to the tasks of determining entity similarity: for example, the similarity of two movies in terms of release date and budget or the similarity of two cities in terms of area and population is currently not captured by the models. Schema level and data level similarity are currently implicitly interwoven, but in particular for knowledge graphs with richer schemas (e.g., YAGO with its type hierarchy of several hundred thousand types), distinguishing embeddings of the schema and data level might become beneficial.

Apart from using vector space embeddings when exploiting LOD data sources, they may also become an interesting technique for improving those sources as such, for example knowledge base completion [40]. Among others, the proposed approach could also be used for link prediction, entity typing, or error detection in knowledge graphs [64], as shown in [45,51]. Similarly to the entity and document modeling, the approach can be extended for entity summarization, which is also an important task when consuming and visualizing large quantities of data [9].

Summarizing, we have shown that it is possible to adapt the technique of word embeddings to RDF graphs, and that those embeddings lead to compact vector representations of entities. We have shown that those vector representations help building approaches which outperform many state of the art tools on various tasks, e.g., data mining with background knowledge or recommender systems. Furthermore, the proposed vector space representations are universal in the sense that they are not task specific, i.e., a vector space embedding for a general graph like DBpedia or Wikidata can be built once and reused for several tasks. As the embeddings used in this paper are publicly available, they are a versatile asset which can be exploited for various knowledge-intensive tasks in future research.

\section{Acknowledgements}

The work presented in this paper has been partly funded by the German Research Foundation (DFG) under grant number PA 2373/1-1 (Mine@LOD), partially funded by the project PON03 PE_00136_1 Digital Services Ecosystem: DSE, and by the Juniorprofessor funding programme of the Ministry of Science, Research and the Arts of the state of BadenWürttemberg (project "Deep semantic models for high-end NLP application"). Jessica Rosati also acknowledges support of I.B.M. Ph.D. fellowship 20152016.

\section{References}

[1] C.C. Aggarwal, A. Hinneburg and D.A. Keim, On the surprising behavior of distance metrics in high dimensional space, in: International Conference on Database Theory, J. Van den Bussche and V. Vianu, eds, Springer Berlin Heidelberg, Berlin, Heidelberg, 2001, pp. 420-434.

[2] N. Aggarwal and P. Buitelaar, Wikipedia-based distributional semantics for entity relatedness, in: Association for the Advancement of Artificial Intelligence (AAAI) Fall Symposium, Washington, DC, USA, 2014.

[3] A. Bellogín, I. Cantador and P. Castells, A comparative study of heterogeneous item recommendations in social systems, Information Sciences 221 (2013), 142-169. doi:10.1016/j.ins. 2012.09.039.

[4] Y. Bengio, R. Ducharme, P. Vincent and C. Janvin, A neural probabilistic language model, Journal of machine learning research 3 (2003), 1137-1155, http://dl.acm.org/citation.cfm? id $=944919.944966$.

[5] C. Bizer, T. Heath and T. Berners-Lee, Linked data - the story so far, International Journal on Semantic Web and Information Systems 5(3) (2009), 1-22, http://ub-madoc.bib. uni-mannheim.de/34762/. doi:10.4018/jswis.2009081901.

[6] A. Bordes, N. Usunier, A. Garcia-Durán, J. Weston and O. Yakhnenko, Translating embeddings for modeling multirelational data, in: Proceedings of the 26th International Conference on Neural Information Processing Systems, NIPS'13, Vol. 2, Curran Associates Inc., USA, 2013, pp. 2787-2795, http://dl.acm.org/citation.cfm?id=2999792.2999923.

[7] S. Brin and L. Page, The anatomy of a large-scale hypertextual web search engine, in: Proceedings of the Seventh International Conference on World Wide Web 7, Elsevier Science Publishers B. V., Amsterdam, The Netherlands, 1998, pp. 107117, http://dl.acm.org/citation.cfm?id=297805.297827. 
[8] R. Burke, Hybrid recommender systems: Survey and experiments, User Modeling and User-Adapted Interaction 12(4) (2002), 331-370. doi: 10.1023/A:1021240730564.

[9] G. Cheng, T. Tran and Y.R. Qu, Relatedness and informativeness-based centrality for entity summarization, in: The Semantic Web-ISWC 2011, L. Aroyo, C. Welty, H. Alani, J. Taylor, A. Bernstein, L. Kagal, N. Noy and E. Blomqvist, eds, Springer Berlin Heidelberg, Berlin, Heidelberg, 2011, pp. 114-129. doi:10.1007/978-3-642-25073-6_8.

[10] W. Cheng, G. Kasneci, T. Graepel, D. Stern and R. Herbrich, Automated feature generation from structured knowledge, in: CIKM, 2011

[11] M. Cochez, P. Ristoski, S.P. Ponzetto and H. Paulheim, Biased graph walks for rdf graph embeddings, in: Proceedings of the 7th International Conference on Web Intelligence, Mining and Semantics, WIMS '17, ACM, New York, NY, USA, 2017, pp. 21:1-21:12, http://doi.acm.org/10.1145/3102254.3102279.

[12] R. Collobert, J. Weston, L. Bottou, M. Karlen, K. Kavukcuoglu and P. Kuksa, Natural language processing (almost) from scratch, Journal of Machine Learning Research 12 (2011), 2493-2537.

[13] C. d'Amato, N. Fanizzi and F. Esposito, A dissimilarity measure for alc concept descriptions, in: Proceedings of the 2006 ACM Symposium on Applied Computing, SAC '06, ACM, New York, NY, USA, 2006, pp. 1695-1699. doi:10.1145/1141277. 1141677.

[14] M. de Gemmis, P. Lops, C. Musto, F. Narducci and G. Semeraro, Semantics-aware content-based recommender systems, in: Recommender Systems Handbook, F. Ricci, L. Rokach and B. Shapira, eds, Springer US, Boston, MA, 2015, pp. 119-159. doi:10.1007/978-1-4899-7637-6_4.

[15] G.K.D. de Vries, A fast approximation of the WeisfeilerLehman graph kernel for rdf data, in: Machine Learning and Knowledge Discovery in Databases, H. Blockeel, K. Kersting, S. Nijssen and F. Železný, eds, Springer Berlin Heidelberg, Berlin, Heidelberg, 2013, pp. 606-621.

[16] G.K.D. De Vries and S. De Rooij, A fast and simple graph kernel for rdf, in: Proceedings of the 2013 International Conference on Data Mining on Linked Data, Vol. 1082, Aachen, Germany, Germany, 2013, pp. 23-34, DMoLD'13, CEURWS.org, http://dl.acm.org/citation.cfm?id=3053776.3053781.

[17] G.K.D. de Vries and S. de Rooij, Substructure counting graph kernels for machine learning from rdf data, Web Semantics: Science, Services and Agents on the World Wide Web $\mathbf{3 5}$ (2015), 71-84. doi:10.1016/j.websem.2015.08.002.

[18] S. Deerwester, S.T. Dumais, G.W. Furnas, T.K. Landauer and R. Harshman, Indexing by latent semantic analysis, Journal of the American Society for Information Science 41(6) (1990), 391-407. doi:10.1002/(SICI)1097-4571(199009)41: 6<391::AID-ASI1>3.0.CO;2-9.

[19] T. Di Noia, R. Mirizzi, V.C. Ostuni and D. Romito, Exploiting the web of data in model-based recommender systems, in: Proceedings of the Sixth ACM Conference on Recommender Systems, RecSys '12, ACM, New York, NY, USA, 2012, pp. 253 256, http://doi.acm.org/10.1145/2365952.2366007.

[20] T. Di Noia, R. Mirizzi, V.C. Ostuni, D. Romito and M. Zanker, Linked open data to support content-based recommender systems, in: Proceedings of the 8th International Conference on Semantic Systems, I-SEMANTICS '12, ACM, New York, NY, USA, 2012, pp. 1-8, http://doi.acm.org/10.1145/2362499. 2362501.
[21] E. Gabrilovich and S. Markovitch, Computing semantic relatedness using Wikipedia-based explicit semantic analysis, in: IJcAI, Vol. 7, 2007, pp. 1606-1611.

[22] Z. Gantner, S. Rendle, C. Freudenthaler and L. SchmidtThieme Mymedialite: A free recommender system library, in: Proceedings of the Fifth ACM Conference on Recommender Systems, RecSys '11, ACM, New York, NY, USA, 2011, pp. 305-308. doi:10.1145/2043932.2043989.

[23] M. Grbovic, V. Radosavljevic, N. Djuric, N. Bhamidipati, J. Savla, V. Bhagwan and D. Sharp, E-commerce in your inbox: Product recommendations at scale, in: Proceedings of the 21th ACM SIGKDD International Conference on Knowledge Discovery and Data Mining, KDD '15, ACM, New York, NY, USA, 2015, pp. 1809-1818. doi:10.1145/2783258.2788627.

[24] A. Grover and J. Leskovec, node2vec: Scalable feature learning for networks, in: Proceedings of the 22nd ACM SIGKDD International Conference on Knowledge Discovery and Data Mining, ACM, 2016, pp. 855-864. doi:10.1145/2939672.2939754.

[25] M.U. Gutmann and A. Hyvärinen, Noise-contrastive estimation of unnormalized statistical models, with applications to natural image statistics, Journal of Machine Learning Research 13 (2012), 307-361.

[26] Z.S. Harris, Mathematical Structures of Language, Wiley, New York, NY, USA, 1968.

[27] S. Hassan and R. Mihalcea, Semantic relatedness using salient semantic analysis, in: Proceedings of the TwentyFifth AAAI Conference on Artificial Intelligence, AAAI'11, AAAI Press, 2011, pp. 884-889, http://dl.acm.org/citation. cfm?id $=2900423.2900564$.

[28] O. Hassanzadeh and M.M. Consens, Linked movie data base, in: Workshop on Linked Data on the Web, 2009.

[29] J. Hoffart, S. Seufert, D.B. Nguyen, M. Theobald and G. Weikum, Kore: Keyphrase overlap relatedness for entity disambiguation, in: Proceedings of the 21st ACM International Conference on Information and Knowledge Management, ACM, 2012, pp. 545-554.

[30] L. Huang, D. Milne, E. Frank and I.H. Witten, Learning a concept-based document similarity measure, Journal of the American Society for Information Science and Technology 63(8) (2012), 1593-1608. doi:10.1002/asi.22689.

[31] Y. Huang, V. Tresp, M. Nickel, A. Rettinger and H.P. Kriegel, A scalable approach for statistical learning in semantic graphs, Semantic Web 5(1) (2014), 5-22.

[32] I. Hulpuş, N. Prangnawarat and C. Hayes, Path-based semantic relatedness on linked data and its use to word and entity disambiguation, in: The Semantic Web-ISWC 2015, M. Arenas, O. Corcho, E. Simperl, M. Strohmaier, M. d'Aquin, K. Srinivas, P. Groth, M. Dumontier, J. Heflin, K. Thirunarayan, K. Thirunarayan and S. Staab, eds, Springer International Publishing, Cham, 2015, pp. 442-457. doi:10.1007/978-3-31925007-6 26

[33] M.A. Khan, G.A. Grimnes and A. Dengel, Two pre-processing operators for improved learning from semanticweb data, in: First RapidMiner Community Meeting and Conference (RCOMM 2010), Vol. 20, 2010.

[34] Y. Koren, Factorization meets the neighborhood: A multifaceted collaborative filtering model, in: Proceedings of the 14th ACM SIGKDD International Conference on Knowledge Discovery and Data Mining, KDD '08, ACM, New York, NY, USA, 2008, pp. 426-434, http://doi.acm.org/10.1145/ 1401890.1401944 
[35] Y. Koren, R. Bell and C. Volinsky, Matrix factorization techniques for recommender systems, Computer 42(8) (2009), 3037. doi:10.1109/MC.2009.263.

[36] S. Kramer, N. Lavrač and P. Flach, Propositionalization approaches to relational data mining, in: Relational Data Mining, Springer, Berlin Heidelberg, 2001, pp. 262-291. doi:10.1007/ 978-3-662-04599-2_11.

[37] M.D. Lee, B. Pincombe and M. Welsh, An empirical evaluation of models of text document similarity, in: Proceedings of the Annual Meeting of the Cognitive Science Society, Vol. 27, 2005.

[38] S. Lee, S. Park, M. Kahng and S.G. Lee, Pathrank: A novel node ranking measure on a heterogeneous graph for recommender systems, in: Proceedings of the 21st ACM International Conference on Information and Knowledge Management, CIKM '12, ACM, New York, NY, USA, 2012, pp. 16371641, http://doi.acm.org/10.1145/2396761.2398488.

[39] J. Lehmann, R. Isele, M. Jakob, A. Jentzsch, D. Kontokostas, P.N. Mendes, S. Hellmann, M. Morsey, P. van Kleef, S. Auer and C. Bizer, DBpedia - A Large-scale, Multilingual Knowledge Base Extracted from Wikipedia, Semantic Web 6(2) (2015), 167-195

[40] Y. Lin, Z. Liu, M. Sun, Y. Liu and X. Zhu, Learning entity and relation embeddings for knowledge graph completion, in: Proceedings of the Twenty-Ninth AAAI Conference on Artificial Intelligence, AAAI'15, AAAI Press, 2015, pp. 2181-2187, http:// dl.acm.org/citation.cfm?id=2886521.2886624.

[41] U. Lösch, S. Bloehdorn and A. Rettinger, Graph kernels for rdf data, in: The Semantic Web: Research and Applications, E. Simperl, P. Cimiano, A. Polleres, O. Corcho and V. Presutti, eds, Springer Berlin Heidelberg, Berlin, Heidelberg, 2012, pp. 134-148. doi:10.1007/978-3-642-30284-8_16.

[42] S.E. Middleton, D.D. Roure and N.R. Shadbolt, OntologyBased Recommender Systems, Springer Berlin Heidelberg, Berlin, Heidelberg, 2009, pp. 779-796. doi:10.1007/978-3540-92673-3_35.

[43] T. Mikolov, K. Chen, G. Corrado and J. Dean, Efficient estimation of word representations in vector space, Computing Research Repository arXiv:abs/1301.3781 (2013), http://dblp. uni-trier.de/db/journals/corr/corr1301.html\#abs-1301-3781.

[44] T. Mikolov, I. Sutskever, K. Chen, G.S. Corrado and J. Dean, Distributed representations of words and phrases and their compositionality, in: Advances in Neural Information Processing Systems, 2013, pp. 3111-3119.

[45] P. Minervini, N. Fanizzi, C. d'Amato and F. Esposito, Scalable learning of entity and predicate embeddings for knowledge graph completion, in: 2015 IEEE 14th International Conference on Machine Learning and Applications (ICMLA), IEEE, 2015, pp. 162-167. doi:10.1109/ICMLA.2015.132.

[46] C. Musto, G. Semeraro, M. De Gemmis and P. Lops, Word embedding techniques for content-based recommender systems: An empirical evaluation, in: RecSys Posters, P. Castells, ed., CEUR Workshop Proceedings, Vol. 1441, 2015.

[47] C. Musto, G. Semeraro, P. Lops and M. de Gemmis, Random Indexing and Negative User Preferences for Enhancing Content-Based Recommender Systems, Springer Berlin Heidelberg, Berlin, Heidelberg, 2011, pp. 270-281. doi:10.1007/ 978-3-642-23014-1_23.

[48] C. Musto, G. Semeraro, P. Lops and M. de Gemmis, Contextual EVSM: A Content-Based Context-Aware Recommendation Framework Based on Distributional Semantics, Springer
Berlin Heidelberg, Berlin, Heidelberg, 2013, pp. 125-136. doi:10.1007/978-3-642-39878-0_12.

[49] J. Mynarz and V. Svátek, Towards a benchmark for LODenhanced knowledge discovery from structured data, in: The Second International Workshop on Knowledge Discovery and Data Mining Meets Linked Open Data, 2013.

[50] V. Narasimha, P. Kappara, R. Ichise and O. Vyas Liddm: A data mining system for linked data, in: Workshop on Linked Data on the Web. CEUR Workshop Proceedings, Vol. 813, 2011, p. 108.

[51] M. Nickel, K. Murphy, V. Tresp and E. Gabrilovich, A review of relational machine learning for knowledge graphs, Proceed ings of the IEEE 104(1) (2016), 11-33. doi:10.1109/JPROC. 2015.2483592.

[52] M. Nickel, L. Rosasco and T. Poggio, Holographic embeddings of knowledge graphs, in: Proceedings of the Thirtieth AAAI Conference on Artificial Intelligence, AAAI'16, AAAI Press, 2016, pp. 1955-1961, http://dl.acm.org/citation.cfm? $\mathrm{id}=3016100.3016172$.

[53] M. Nickel, V. Tresp and H.P. Kriegel, A three-way model for collective learning on multi-relational data, in: Proceedings of the 28th International Conference on Machine Learning (ICML-11), 2011, pp. 809-816.

[54] X. Ning and G. Karypis, SLIM: sparse linear methods for top-n recommender systems, in: 11th IEEE International Conference on Data Mining, ICDM 2011, Vancouver, BC, Canada, December 11-14, 2011, 2011, pp. 497-506. doi:10.1109/ICDM.2011. 134.

[55] X. Ning and G. Karypis, Sparse linear methods with side information for top-n recommendations, in: Proceedings of the Sixth ACM Conference on Recommender Systems, RecSys '12, ACM, New York, NY, USA, 2012, pp. 155-162. doi:10.1145/ 2365952.2365983.

[56] T.D. Noia, V.C. Ostuni, J. Rosati, P. Tomeo, E.D. Sciascio, R. Mirizzi and C. Bartolini, Building a relatedness graph from linked open data: A case study in the it domain, Expert Systems with Applications 44 (2016), 354-366, http://www. sciencedirect.com/science/article/pii/S0957417415005941. doi:10.1016/j.eswa.2015.08.038.

[57] T.D. Noia, V.C. Ostuni, P. Tomeo and E.D. Sciascio Sprank: Semantic path-based ranking for top-n recommendations using linked open data, ACM Trans. Intell. Syst. Technol. 8(1) (2016), 9:1-9:34. doi:10.1145/2899005.

[58] B.P. Nunes, B. Fetahu, R. Kawase, S. Dietze, M.A. Casanova and D. Maynard, Interlinking Documents Based on Semantic Graphs with an Application, Springer International Publishing, Cham, 2015, pp. 139-155. doi:10.1007/978-3-31913545-8_9.

[59] S. Oramas, V.C. Ostuni, T.D. Noia, X. Serra and E.D. Sciascio, Sound and music recommendation with knowledge graphs, ACM Trans. Intell. Syst. Technol. 8(2) (2016), 21:1-21:21. doi:10.1145/2926718.

[60] V.C. Ostuni, T. Di Noia, E. Di Sciascio and R. Mirizzi, Top$\mathrm{n}$ recommendations from implicit feedback leveraging linked open data, in: Proceedings of the 7th ACM Conference on Recommender Systems, RecSys '13, ACM, New York, NY, USA, 2013, pp. 85-92. doi:10.1145/2507157.2507172.

[61] M.G. Ozsoy, From word embeddings to item recommendation, 2016, arXiv preprint arXiv:1601.01356. 
[62] C. Paul, A. Rettinger, A. Mogadala, C.A. Knoblock and P. Szekely, Efficient graph-based document similarity, in: Proceedings of the 13th International Conference on the Semantic Web. Latest Advances and New Domains, Vol. 9678, SpringerVerlag, Berlin, Heidelberg, 2016, pp. 334-349. doi:10.1007/ 978-3-319-34129-3 21.

[63] H. Paulheim, Exploiting linked open data as background knowledge in data mining, in: Proceedings of the 2013 International Conference on Data Mining on Linked Data, DMoLD'13, Vol. 1082, Aachen, Germany, Germany, 2013 , pp. 1-10, CEUR-WS.org, http://dl.acm.org/citation.cfm? id $=3053776.3053778$.

[64] H. Paulheim, Knowledge graph refinement: A survey of approaches and evaluation methods, Semantic Web 8(3) (2016), 489-508. doi:10.3233/SW-160218.

[65] H. Paulheim and J. Fümkranz, Unsupervised generation of data mining features from linked open data, in: Proceedings of the 2nd International Conference on Web Intelligence, Mining and Semantics, ACM, 2012, p. 31.

[66] H. Paulheim, P. Ristoski, E. Mitichkin and C. Bizer, Data mining with background knowledge from the Web, in: RapidMiner World, 2014, pp. 1-14.

[67] B. Perozzi, R. Al-Rfou and S. Skiena Deepwalk: Online learning of social representations, in: Proceedings of the 20th ACM SIGKDD International Conference on Knowledge Discovery and Data Mining, ACM, 2014, pp. 701-710.

[68] S. Rendle, Factorization machines with libfm, ACM Transactions Intelligent Systems and Technology 3(3) (2012), 57:157:22. doi:10.1145/2168752.2168771.

[69] S. Rendle, C. Freudenthaler, Z. Gantner and L. SchmidtThieme Bpr: Bayesian personalized ranking from implicit feedback, in: Proceedings of the Twenty-Fifth Conference on Uncertainty in Artificial Intelligence, Arlington, Virginia, United States, 2009, pp. 452-461, UAI '09, http://dl.acm.org/ citation.cfm?id=1795114.1795167.

[70] F. Ricci, L. Rokach, B. Shapira and P.B. Kantor, Recommender Systems Handbook, 2nd edn, Springer-Verlag New York, Inc., New York, NY, USA, 2015.

[71] D. Ringler and H. Paulheim, One knowledge graph to rule them all? Analyzing the differences between dbpedia, yago, wikidata and co, in: 40th German Conference on Artificial Intelligence, 2017.

[72] P. Ristoski, C. Bizer and H. Paulheim, Mining the web of linked data with rapidminer, Web Semantics: Science, Services and Agents on the World Wide Web 35 (2015), 142-151. doi:10. 1016/j.websem.2015.06.004.

[73] P. Ristoski, G.K.D. de Vries and H. Paulheim, A collection of benchmark datasets for systematic evaluations of machine learning on the semantic Web, in: The Semantic Web - ISWC 2016, P. Groth, E. Simperl, A. Gray, M. Sabou, M. Krötzsch, F. Lecue, F. Flöck and Y. Gil, eds, Springer International Publishing, Cham, 2016, pp. 186-194. doi:10.1007/978-3-31946547-0_20.

[74] P. Ristoski, E.L. Mencía and H. Paulheim, A hybrid multistrategy recommender system using linked open data, in: $\mathrm{Se}$ mantic Web Evaluation Challenge, Springer, 2014, pp. 150156.

[75] P. Ristoski and H. Paulheim, A comparison of propositionalization strategies for creating features from linked open data, in: Proceedings of the 1st International Conference on Linked Data for Knowledge Discovery, Vol. 1232, Aachen, Germany,
Germany, 2014, pp. 1-11, LD4KD'14, CEUR-WS.org, http:// dl.acm.org/citation.cfm?id=3053827.3053828.

[76] P. Ristoski and H. Paulheim, Rdf2vec: Rdf graph embeddings for data mining, in: International Semantic Web Conference, Springer, 2016, pp. 498-514.

[77] P. Ristoski and H. Paulheim, Semantic web in data mining and knowledge discovery, Web Semant. 36(C) (2016), 1-22. doi:10. 1016/j.websem.2016.01.001.

[78] J. Rosati, P. Ristoski, T.D. Noia, R.D. Leone and H. Paulheim, Rdf graph embeddings for content-based recommender systems, in: Proceedings of the 3rd Workshop on New Trends in Content-Based Recommender Systems (CBRecSys 2016), 2016.

[79] M. Sahlgren, An introduction to random indexing, in: Methods and Applications of Semantic Indexing Workshop at the 7th International Conference on Terminology and Knowledge Engineering, TKE 2005, 2005.

[80] R. Salakhutdinov and A. Mnih, Bayesian probabilistic matrix factorization using Markov chain Monte Carlo, in: Proceedings of the 25th International Conference on Machine Learning, ICML '08, ACM, New York, NY, USA, 2008, pp. 880887. doi:10.1145/1390156.1390267.

[81] M. Schmachtenberg, C. Bizer and H. Paulheim, Adoption of the linked data best practices in different topical domains, in: The Semantic Web - ISWC 2014, P. Mika, T. Tudorache, A. Bernstein, C. Welty, C. Knoblock, D. Vrandečić, P. Groth, N. Noy, K. Janowicz and C. Goble, eds, Springer International Publishing, Cham, 2014, pp. 245-260.

[82] M. Schuhmacher and S.P. Ponzetto, Knowledge-based graph document modeling, in: Proceedings of the 7th ACM International Conference on Web Search and Data Mining, ACM, 2014, pp. 543-552. doi:10.1145/2556195.2556250.

[83] G. Semeraro, P. Lops, P. Basile and M. de Gemmis, Knowledge infusion into content-based recommender systems, in: Proceedings of the Third ACM Conference on Recommender Systems, RecSys '09, ACM, New York, NY, USA, 2009, pp. 301304. doi:10.1145/1639714.1639773.

[84] N. Shervashidze, P. Schweitzer, E.J. Van Leeuwen, K. Mehlhorn and K.M. Borgwardt, Weisfeiler-Lehman graph kernels, The Journal of Machine Learning Research 12 (2011), 2539-2561.

[85] R. Socher, D. Chen, C.D. Manning and A. Ng, Reasoning with neural tensor networks for knowledge base completion, in: Advances in Neural Information Processing Systems, 2013, pp. 926-934.

[86] N. Srebro, J.D.M. Rennie and T.S. Jaakola, Maximum-margin matrix factorization, in: Advances in Neural Information Processing Systems, Vol. 17, MIT Press, 2005, pp. 1329-1336.

[87] H. Steck, Evaluation of recommendations: Rating-prediction and ranking, in: Proceedings of the 7th ACM Conference on Recommender Systems, RecSys '13, ACM, New York, NY, USA, 2013, pp. 213-220. doi:10.1145/2507157.2507160.

[88] R. Thiagarajan, G. Manjunath and M. Stumptner, Computing semantic similarity using ontologies, Technical report HPL2008-87, HP Laboratories, 2008.

[89] T. Trouillon, J. Welbl, S. Riedel, E. Gaussier and G. Bouchard, Complex embeddings for simple link prediction, in: Proceedings of the 33rd International Conference on International Conference on Machine Learning, ICML'16, Vol. 48, 2016, pp. 2071-2080, JMLR.org http://dl.acm.org/citation. cfm?id=3045390.3045609. 
[94] D. Widdows, Orthogonal negation in vector spaces for modelling word-meanings and document retrieval, in: Proceedings of the 41st Annual Meeting on Association for Computational Linguistics - Volume 1, ACL '03, Association for Computational Linguistics, Stroudsburg, PA, USA, 2003, pp. 136-143. doi: 10.3115/1075096.1075114.

[95] P. Yanardag and S. Vishwanathan, Deep graph kernels, in: Proceedings of the 21th ACM SIGKDD International Conference on Knowledge Discovery and Data Mining, ACM, 2015, pp. 1365-1374. doi:10.1145/2783258.2783417.

[96] X. Yu, X. Ren, Y. Sun, Q. Gu, B. Sturt, U. Khandelwal, B. Norick and J. Han, Personalized entity recommendation: A heterogeneous information network approach, in: Proceedings of the 7th ACM International Conference on Web Search and Data Mining, WSDM '14, ACM, New York, NY, USA, 2014, pp. 283-292. doi:10.1145/2556195.2556259.
52 53 54 56 57 58 59 60 